\title{
Infrared Classification and Luminosities For Dusty AGN and the Most Luminous Quasars
}

\author{
Daniel Weedman ${ }^{1}$, Lusine Sargsyan ${ }^{1}$, Vianney Lebouteiller ${ }^{2}$, James Houck ${ }^{1}$, and Donald \\ Barry $^{1}$
}

\begin{abstract}
Mid-infrared spectroscopic measurements from the Infrared Spectrometer on Spitzer (IRS) are given for 125 hard X-ray AGN (14-195 keV) from the Swift Burst Alert Telescope sample and for 32 AGN with black hole masses from reverberation mapping. The $9.7 \mu \mathrm{m}$ silicate feature in emission or absorption defines an infrared AGN classification describing whether AGN are observed through dust clouds, indicating that $55 \%$ of the BAT AGN are observed through dust. The mid-infrared dust continuum luminosity is shown to be an excellent indicator of intrinsic AGN luminosity, scaling closely with the hard X-ray luminosity, log $\nu L_{\nu}(7.8 \mu \mathrm{m}) / L(\mathrm{X})=-0.31 \pm 0.35$ and independent of classification determined from silicate emission or absorption. Dust luminosity scales closely with black hole mass, $\log \nu L_{\nu}(7.8 \mu \mathrm{m})=(37.2 \pm 0.5)+0.87 \log$ BHM for luminosity in erg $\mathrm{s}^{-1}$ and $\mathrm{BHM}$ in $\mathrm{M}_{\odot}$. The 100 most luminous type 1 quasars as measured in $\nu L_{\nu}(7.8 \mu \mathrm{m})$ are found by comparing Sloan Digital Sky Survey optically discovered quasars with photometry at $22 \mu \mathrm{m}$ from the Wide-Field Infrared Survey Explorer, scaled to rest frame $7.8 \mu \mathrm{m}$ using an empirical template determined from IRS spectra. The most luminous SDSS/WISE quasars have the same maximum infrared luminosities for all $1.5<\mathrm{z}<5$, reaching total infrared luminosity $L_{I R}=10^{14.4} \mathrm{~L}_{\odot}$. Comparing with Dust Obscured Galaxies from Spitzer and WISE surveys, we find no evidence of hyperluminous obscured quasars whose maximum infrared luminosities exceed the maximum infrared luminosities of optically discovered quasars. Bolometric luminosities $L_{b o l}$ estimated from rest frame optical or ultraviolet luminosities are compared to $L_{I R}$. For the local AGN, the median $\log L_{I R} / L_{b o l}=-0.35$, consistent with a covering factor of $45 \%$ for the absorbing dust clouds. For the SDSS/WISE quasars, the median log $L_{I R} / L_{b o l}$ $=0.1$, with extremes indicating that ultraviolet-derived $L_{b o l}$ can be seriously underestimated even for type 1 quasars.
\end{abstract}

\footnotetext{
${ }^{1}$ Astronomy Department, Cornell University, Ithaca, NY 14853; dweedman@isc.astro.cornell.edu

${ }^{2}$ Laboratoire AIM, CEA/DSM-CNRS-Universite Paris Diderot, DAPNIA/Service d'Astrophysique, Saclay, France
} 
Subject headings: infrared: galaxies — galaxies: starburst — galaxies: active galaxies: distances and redshifts - galaxies: evolution-- quasars: general

\section{Introduction}

Observational understanding of the initial assembly of galaxies within the early Universe arises by tracing the luminosities and characteristics of galaxies as a function of redshift. The astrophysical questions are fundamental: How and when did the first generation of stars form and assemble into galaxies? When and how did the supermassive black holes (SMBH) within quasars or active galactic nuclei (AGNs) develop? How massive can be early galaxies and SMBH? What is the connection between initial star formation and AGN?

A specific observational challenge is to trace these formation processes to their highest redshifts. For luminous quasars and luminous star formation, this has been most successful using optically discovered sources whose luminosities seem to peak at $2 \lesssim z \leqslant 3$ when observed in the rest frame ultraviolet (e.g. Madau et al. 1998; Fan et al. 2004; Croom et al. 2004; Reddy and Steidel 2009). It is also known, however, that there are many luminous but dusty sources whose dust obscures the primary optical, ultraviolet and even X-ray sources of luminosity. The existence of such dusty objects means that a census of the universe derived only from optical observations must be incomplete.

It is known, for example, that the most luminous galaxies $\left(L>10^{13} \mathrm{~L}_{\odot}\right)$ in the local universe are the Ultraluminous Infrared Galaxies (ULIRGs, e.g. Soifer, Neugebauer and Houck 1987, Sanders and Mirabel 1996) whose luminosity arises from reemission by dust at infrared wavelengths of electromagnetic radiation initially generated at much shorter wavelengths. Surveys in the submillimeter initially discovered individual, optically obscured, dusty sources at redshifts z $\gtrsim 2$ (Chapman et al. 2005). Various observing programs to understand optically faint infrared sources using spectra from the Spitzer Infrared Spectrometer (IRS; Houck et al. 2004) found luminous ULIRGS to redshifts z $\sim 3$ (e.g. Houck et al. 2005; Yan et al. 2007; Sajina et al. 2007; Weedman and Houck 2009). This Spitzer-discovered population of high redshift ULIRGs has large infrared to optical flux ratios $\left[f_{\nu}(24 \mu \mathrm{m})>1\right.$ mJy and $R>24$ ] and has been labeled "dust obscured galaxies" (DOGS; Dey et al. 2008). DOGs are the high redshift, most luminous examples of ULIRGs.

The requirement for a high redshift population of AGN with substantial multiwavelength extinction was initially demonstrated by the spectrum of the X-ray background, requiring a harder spectrum than could be explained by the population of unobscured type 1 AGN and quasars (Setti and Woltjer 1989). This harder spectrum was explained by a population 
of "Compton-thick" AGN having sufficient column densities of gas $\left(\gtrsim 10^{24} \mathrm{~cm}^{-2}\right)$ to absorb soft X-rays (e.g. Fabian 1999; Gilli et al. 2007). Various studies confirmed the presence of a population of obscured AGN (usually considered as type 2 AGN) that outnumbers unobscured AGN (type 1 AGN) by a factor of a few (Maiolino and Rieke 1995; Willott et al. 2000; Alexander et al. 2003; Martinez-Sansigre et al. 2006; Hickox et al. 2007).

The most luminous DOGS also have multiwavelength spectral characteristics of AGN, including X-ray luminosity, "power law" near infrared spectra without indication of stellar spectral features, and mid-infrared spectra without the polycyclic aromatic hydrocarbon (PAH) features that characterise starbursts (e.g. Brand et al. 2006; Donley et al. 2007; Fiore et al. 2008; Bussmann et al. 2009). A natural conclusion is that these sources are a population of AGN that represents those type 2 AGN which are most extreme in obscuration and luminosity .

The major advantage of using infrared luminosities to characterise sources is that all AGN can be compared consistently without the uncertain extinction corrections necessary for luminosity measures at shorter wavelengths. For example, dust extinction at $0.2 \mu \mathrm{m}$ is a factor of $\sim 100$ greater than at $20 \mu \mathrm{m}$ (Draine 2003a, b). With the completion of the Spitzer mission and the full archive of spectra from the IRS, many previously defined samples of AGN and quasars can now be uniformly measured in the mid-infrared from $\sim 5 \mu \mathrm{m}$ to $\sim 35$ $\mu \mathrm{m}$. Thousands of low resolution spectra are available in the public archive "The Cornell Atlas of Spitzer/IRS Spectra" (CASSIS; Lebouteiller et al. 2011ㄱ), a database of IRS spectra extracted in an optimal and consistent manner.

In the present paper, we describe and calibrate an infrared AGN classification, measure infrared luminosities, and compare to virial black hole masses and optically-derived bolometric luminosities by analyzing CASSIS spectra for the following samples:

1. The uniform sample of AGN in Tueller et al. (2010) discovered because of high energy X-rays in the all sky sample from the Burst Alert Telescope (BAT) on the Swift Small Explorer (Gehrels et al. 2004); 125 of these have IRS low resolution spectra.

2. The AGN with the most accurate determinations of virial black hole masses, using the reverberation mapping technique to determine scale size of the broad line region (Peterson et al. 2004); 32 have IRS low resolution spectra (many of these are also in the BAT sample).

3. The 100 type 1 quasars most luminous in the mid-infrared $\left[\nu L_{\nu}(7.8 \mu \mathrm{m})\right]$; these are found by combining an empirical spectral template determined with the IRS for type 1 AGN and

\footnotetext{
${ }^{1}$ http://cassis.astro.cornell.edu/atlas; CASSIS is a product of the Infrared Science Center at Cornell University.
} 
quasars with photometry at $22 \mu \mathrm{m}$ from the Wide-Field Infrared Survey Explorer (WISE, Wright et al. 2010) for optically discovered quasars in the Sloan Digital Sky Survey (SDSS; Gunn et al. 1998, Schneider et al. 2010).

The results give consistent measures of dust luminosity for local AGN and for the most luminous type 1 quasars to $\mathrm{z}=5$. Results are compared to the infrared luminosities for the most luminous DOGs discovered both by Spitzer and by WISE.

\section{IR Spectral Classification and Dust Luminosities}

The mid-infrared spectra of AGN are dominated by the continuum emission from dust and show direct evidence of either dust emission or absorption, revealed primarily by the $9.7 \mu \mathrm{m}$ silicate feature (Figure 1). The presence of silicate absorption means there is cooler dust between the observer and the hotter dust responsible for the infrared continuum. The infrared spectra of absorbed AGN are consistent with observing an AGN behind dust clouds, with dust on the inner side of the absorbing clouds heated by the AGN (e.g. Imanishi et al. 2007). When classifiable optically, these AGN are most often type 2 (Hao et al. 2007) but often are not classifiable optically because of strong extinction of the AGN.

By contrast, type $1 \mathrm{AGN}$ and quasars generally show silicate emission (e.g. Hao et al. 2005, 2007), which indicates that the hot dust is observed without intervening cool dust. This arises when the circumnuclear dust clouds are observed sufficiently face on that the heated side of the clouds is directly observed so silicate emission is seen. While orientation effects alone may not explain all the spectroscopic differences among AGN, the success of dusty torus models in explaining all AGN types indicates that circumnuclear dust is a general characteristic of AGN (Shi et al. 2006; Ramos-Almeida et al. 2011).

The geometrical dust distributions required to explain silicate absorption and emission observed in IRS spectra, and how this relates to optical classifications, have been carefully

discussed (Levenson et al. 2007; Sirocky et al. 2008; Thompson et al. 2009; Elitzur 2012). The essential conclusions are that the only geometries ruled out by observations are spherically symmetric and uniform dust screens. Silicate absorption can take place through either uniform screens or clumpy dust distributions, but silicate emission cannot be observed unless some lines of sight do not pass through dust which is cooler than the emitting dust. Presence of silicate emission requires, therefore, either toroidal distributions of dust or spherical distributions of clumpy dust so that there are some lines of sight where an observer can see directly the emitting dust heated by the AGN.

Detailed models of dust distributions including optical depth effects for absorbing clouds 
are not completely consistent with the simplest "unified theory" whereby all AGN are fundamentally similar but differ in appearance from type 1 to type 2 only because of orientation to the observer. A distribution of dust geometries is required, and the most extreme examples derive from the most extreme geometries with the largest covering factors for the dust (Elitzur 2012). For our purposes in the present paper, the most important question is how well the infrared luminosity from dust, $L_{I R}$, measures the total intrinsic luminosity, $L_{b o l}$, generated by the AGN. The ratio $L_{I R} / L_{b o l}$ is a measure of the covering factor of the dust clouds which absorb the primary radiation from $L_{b o l}$ and reemit as $L_{I R}$.

Although the presence of silicate absorption or emission in infrared spectra generally correlates with type 2 and type 1 optical classification, the qualitative nature of optical classifications and the dependence of optical parameters on extinction corrections provides a motive for classification based only on the infrared silicate spectrum. In the present paper, we quantitatively classify sources depending on the measured strength of the silicate absorption or emission. We call sources "obscured AGN" if they have silicate absorption and "unobscured AGN" if they have silicate emission. This single parameter AGN classification based on silicate strength is described below.

\subsection{AGN Classification by Silicate Strength and Mid-Infrared Luminosities}

The infrared classification and luminosity parameters which we utilize are illustrated in Figure 1. The classification is a measure of the height or depth of the silicate feature measured at $10 \mu \mathrm{m}$. As illustrated in Figure 1, this measure of silicate strength assumes an extrapolated dust continuum between $7.8 \mu \mathrm{m}$ and $13 \mu \mathrm{m}$, and the silicate feature is measured relative to this continuum. The strength of the silicate feature is defined as $f_{\nu}(10$ $\mu \mathrm{m}$ observed $) / f_{\nu}(10 \mu \mathrm{m}$ continuum $)$, for $f_{\nu}(10 \mu \mathrm{m}$ continuum $)$ extrapolated linearly between $f_{\nu}(7.8 \mu \mathrm{m})$ and $f_{\nu}(13 \mu \mathrm{m})$, as shown.

This parameter is designed primarily to allow estimation of silicate absorption in DOGs at $\mathrm{z} \gtrsim 2$, for which IRS spectra have limited wavelength coverage and poor signal to noise $(\mathrm{S} / \mathrm{N})$. Various alternatives for measuring silicate strength when longer, reliable continuum baselines can be used are discussed by Spoon et al. (2007) and Sirocky et al. (2008). Our measurement is similar to one of the continuum fits they illustrate, except that we do not express the silicate strength as an optical depth, and we always measure at a single wavelength instead of seeking the wavelength where the feature is strongest.

The observed mid-infrared luminosity is determined as $\nu L_{\nu}(7.8 \mu \mathrm{m})$ (rest frame). This luminosity parameter is chosen because it is the most reliable flux measurement for dusty 


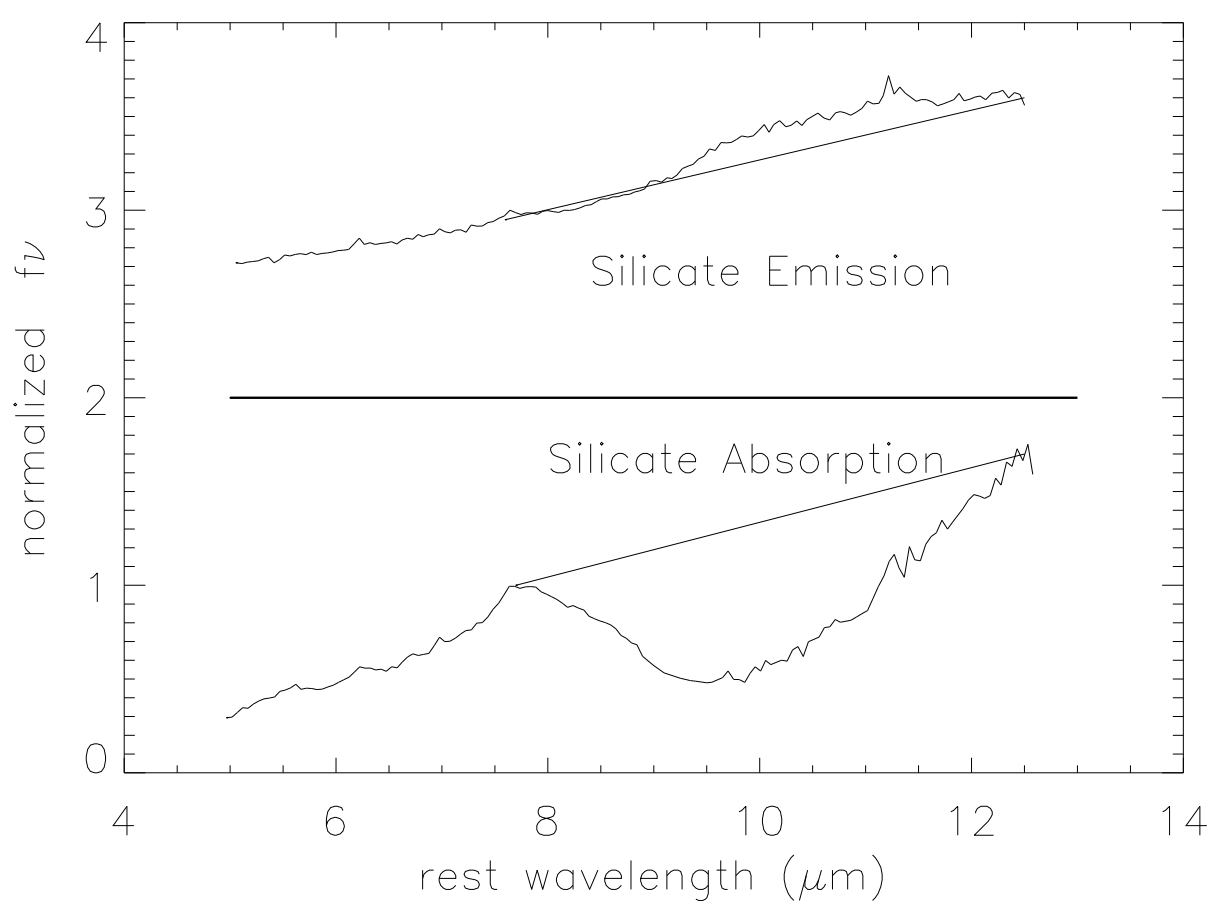

Fig. 1.- Average of silicate emission AGN and silicate absorption AGN from the IRS spectra in Sargsyan et al. (2011), showing the $9.7 \mu \mathrm{m}$ silicate feature. The strength of the silicate feature is defined as the observed flux density at $10 \mu \mathrm{m}$ compared to the extrapolated continuum between $7.8 \mu \mathrm{m}$ and $13 \mu \mathrm{m}$, shown by the solid lines. Spectra shown are normalized to $f_{\nu}(7.8 \mu \mathrm{m})=1 \mathrm{mJy}$; zero levels are offset by $2 \mathrm{mJy}$ so horizontal line is zero level for upper spectrum. With this definition, the silicate strength of the upper spectrum is 1.12 and of the lower spectrum is 0.42 . Mid-infrared luminosities are defined using flux $\nu f_{\nu}(7.8$ $\mu \mathrm{m})$ at the observed wavelength corresponding to rest frame $7.8 \mu \mathrm{m}$. 
sources, especially faint sources at $\mathrm{z} \gtrsim 2$ with IRS spectra (summarized by Weedman and Houck (2009)), because this wavelength is a localized continuum maximum between absorptions on either side for heavily obscured sources (as seen in Figure 1). This luminosity parameter is especially important for highly obscured, optically faint DOGs when optical spectra cannot be obtained. For silicate emission sources, such as optically discovered quasars, measuring $\nu L_{\nu}(7.8 \mu \mathrm{m})$ allows an unambiguous luminosity comparison with the obscured sources.

The greatest uncertainties in measuring $\nu L_{\nu}(7.8 \mu \mathrm{m})$ or the silicate strength in this way arise when PAH features from a starburst are present, because the flux density at $7.8 \mu \mathrm{m}$ is not then a purely continuum measurement but also includes the peak of the $7.7 \mu \mathrm{m} \mathrm{PAH}$ feature. For this reason, we do not measure silicate strength or $\nu L_{\nu}(7.8 \mu \mathrm{m})$ for any source with $\operatorname{EW}(6.2 \mu \mathrm{m} \mathrm{PAH})>0.05 \mu \mathrm{m}$, as discussed below in section 2.2. As can be seen by examining individual spectra in CASSIS, uncertainties in the measure of silicate strength are all in the direction that silicate emission would be underestimated and silicate absorption overestimated. This is partly because any PAH contribution would artificially raise the assumed continuum at $7.8 \mu \mathrm{m}$ and partly because the peak of silicate emission moves to wavelengths longer than $10 \mu \mathrm{m}$ for sources with strong silicate emission.

\subsection{Infrared Classification for the BAT AGN Sample}

The infrared AGN classification derived from silicate strength is now demonstrated for a uniform and independent AGN sample using comparisons between CASSIS infrared spectra and characteristics of the uniform sample of AGN defined by hard X-rays (14-195 keV) from the Swift BAT all sky survey (Tueller et al. 2010). As those authors explain, this is an unbiased AGN sample discovered without regard to optical classification, using only the presence of hard X-rays. Consequently, it is an excellent sample for objective tests of the infrared classification criteria. Of the 234 sources listed in the BAT sample having "Sy" or "quasar" optical classifications (Table 5 of Tueller et al.), 125 have IRS spectra in CASSIS. Results for these AGN are summarized in Table 1. 
Table 1. Observed Properties of BAT AGN

\begin{tabular}{|c|c|c|c|c|c|c|c|c|c|}
\hline No. & $\mathrm{Name}^{\mathrm{a}}$ & $\mathrm{AOR}^{\mathrm{b}}$ & $\begin{array}{c}\operatorname{flux}(\mathrm{X})^{\mathrm{c}} \\
10^{-11}\end{array}$ & $\begin{array}{c}L(\mathrm{X})^{\mathrm{d}} \\
\log \operatorname{erg~s}^{-1}\end{array}$ & $\begin{array}{c}\mathrm{f}([\mathrm{NeIII}])^{\mathrm{e}} \\
10^{-21}\end{array}$ & $\begin{array}{l}\mathrm{f}([\mathrm{OIV}])^{\mathrm{f}} \\
10^{-21}\end{array}$ & $\begin{array}{c}\mathrm{EW}(6.2 \mu \mathrm{m})^{\mathrm{g}} \\
\mu \mathrm{m}\end{array}$ & $\begin{array}{c}f_{\nu}(7.8 \mu \mathrm{m})^{\mathrm{h}} \\
\mathrm{mJy}\end{array}$ & silicate $^{\mathrm{i}}$ \\
\hline 1 & Mrk 335 & 14448128 & 2.47 & 43.57 & $\cdots$ & $\cdots$ & $<0.01$ & 128 & 1.08 \\
\hline 2 & Mrk 1501 & 4857088,14188544 & 4.10 & 44.91 & 3.9 & 8.4 & $<0.01$ & 53 & 1.0 \\
\hline 3 & NGC $235 \mathrm{~A}$ & 20342016 & 4.12 & 43.67 & 8.8 & 23.1 & 0.084 & 79 & . \\
\hline 4 & Mrk 348 & 17957376 & 13.66 & 43.84 & 23.6 & 18.5 & $<0.01$ & 165 & 0.76 \\
\hline 5 & Mrk 1148 & 14189056 & 2.80 & 44.44 & 0.68 & 0.35 & $<0.01$ & 10.7 & 1.16 \\
\hline 6 & Mrk 352 & 26482944 & 4.16 & 43.31 & $\cdots$ & $\cdots$ & 0.03 & 6.2 & 1.36 \\
\hline 7 & 3C 033 & 11295744 & 4.06 & 44.54 & 3.59 & 6.39 & $<0.01$ & 24 & 0.78 \\
\hline 8 & Fairall9 & 18505984,28720896 & 5.07 & 44.42 & 3.9 & 5.1 & $<0.01$ & 197 & 1.11 \\
\hline 9 & NGC 526a & 18525184,4867328 & 5.96 & 43.69 & 10.1 & 17.4 & $<0.01$ & 98 & 0.9 \\
\hline 10 & NGC 612 & 18945280 & 5.35 & 44.04 & 3.1 & 5.2 & 0.22 & 70 & $\cdots$ \\
\hline 11 & ESO 297 & 18944768 & 7.37 & 44.03 & 2.6 & 3.3 & 0.036 & 35 & 0.72 \\
\hline 12 & NGC 788 & 18944512 & 9.33 & 43.59 & 13.9 & 18 & $<0.01$ & 61 & 0.77 \\
\hline 13 & Mrk 1018 & 15076096 & 3.61 & 44.18 & 1.6 & 1.8 & $<0.01$ & 40 & 1.19 \\
\hline 14 & IC 1816 & 26485248 & 2.58 & 43.22 & 19.5 & 16.7 & 0.045 & 50 & 0.64 \\
\hline 15 & NGC 973 & 26485760 & 3.09 & 43.26 & 9.0 & 20 & $<0.01$ & 56 & 0.40 \\
\hline 16 & NGC 985 & 13022720 & 3.45 & 44.17 & 13 & 22 & $<0.01$ & 100 & 0.95 \\
\hline 17 & ESO 198-024 & 26491904 & 4.98 & 44.38 & $\ldots$ & $\ldots$ & $<0.01$ & 22 & 1.0 \\
\hline 18 & NGC 1052 & 18258688 & 3.75 & 42.32 & 14.3 & 6.3 & $<0.01$ & 70 & 0.94 \\
\hline 19 & ESO 417-G006 & 26486784 & 3.06 & 43.26 & 2.6 & 3.9 & $<0.01$ & 9.3 & 0.79 \\
\hline 20 & NGC 1275 & 3753984 & 6.85 & 43.68 & 27 & $<5$ & $<0.01$ & 232 & 1.0 \\
\hline 21 & NGC 1365 & 9075200,8767232 & 7.19 & 42.68 & 74 & 131 & 0.059 & 504 & $\cdots$ \\
\hline 22 & ESO 548 & 18943744 & 4.57 & 43.33 & 2.8 & 2.6 & $<0.01$ & 92 & 1.21 \\
\hline 23 & 2MASX J03502377 & 26496512 & 3.09 & 43.98 & 2.0 & 1.1 & 0.49 & 18 & $\cdots$ \\
\hline 24 & ESO 549 & 26489600 & 2.65 & 43.62 & 4.5 & 9.5 & 0.23 & 113 & $\ldots$ \\
\hline 25 & ESO 157 G23 & 26498816 & 3.02 & 44.13 & 2.2 & 4.3 & $<0.01$ & 22.7 & 1.0 \\
\hline 26 & $3 \mathrm{C} 120$ & 18505216,4847360 & 11.89 & 44.48 & 26.9 & 91 & $<0.01$ & 138 & 1.0 \\
\hline 27 & MCG-02-12-050 & 26498560 & 2.11 & 43.81 & 1.9 & 2.5 & $<0.01$ & 30 & 1.0 \\
\hline
\end{tabular}


Table 1-Continued

\begin{tabular}{|c|c|c|c|c|c|c|c|c|c|}
\hline No. & Name $^{\mathrm{a}}$ & $\mathrm{AOR}^{\mathrm{b}}$ & $\begin{array}{c}\operatorname{flux}(\mathrm{X})^{\mathrm{c}} \\
10^{-11}\end{array}$ & $\begin{array}{c}L(\mathrm{X})^{\mathrm{d}} \\
\log \operatorname{erg~s}^{-1}\end{array}$ & $\begin{array}{c}\mathrm{f}([\mathrm{NeIII}])^{\mathrm{e}} \\
10^{-21}\end{array}$ & $\begin{array}{c}\mathrm{f}([\mathrm{OIV}])^{\mathrm{f}} \\
10^{-21}\end{array}$ & $\begin{array}{c}\mathrm{EW}(6.2 \mu \mathrm{m})^{\mathrm{g}} \\
\mu \mathrm{m}\end{array}$ & $\begin{array}{l}f_{\nu}(7.8 \mu \mathrm{m})^{\mathrm{h}} \\
\mathrm{mJy}\end{array}$ & silicate $^{\mathrm{i}}$ \\
\hline 28 & CGCG 420 & 26482176 & 4.08 & 43.91 & 5.7 & 9.9 & $<0.01$ & 171 & 1.0 \\
\hline 29 & 2MASX J05054575 & 26490368 & 7.16 & 44.31 & 2.5 & 1.8 & $<0.01$ & 36 & 0.83 \\
\hline 30 & Ark 120 & 18941440 & 7.08 & 44.23 & 2.6 & 3.0 & $<0.01$ & 177 & 1.19 \\
\hline 31 & PictorA & 4673792 & 3.78 & 44.03 & 1.9 & $<0.5$ & $<0.01$ & 31 & 1.27 \\
\hline 32 & IRAS 05218 & 26497536 & 2.50 & 44.15 & 7.3 & 15.7 & $<0.01$ & 73 & 0.95 \\
\hline 33 & NGC 2110 & 4851456,18509312 & 35.01 & 43.67 & 51 & 38 & 0.013 & 155 & 1.0 \\
\hline 34 & 2MASX J05580206 & 18943232 & 3.99 & 44.02 & 4.7 & 4.5 & $<0.01$ & 305 & 0.79 \\
\hline 35 & ESO 005-G004 & 18947328 & 4.48 & 42.59 & 9.3 & $<3.0$ & 0.06 & 79 & 0.21 \\
\hline 36 & Mrk 3 & 3753472 & 15.65 & 43.81 & 188 & 178 & $<0.01$ & 235 & 0.63 \\
\hline 37 & ESO 426 & 26495232 & 2.72 & 43.49 & 3.0 & 12.8 & $<0.01$ & 43 & 0.86 \\
\hline 38 & UGC 03601 & 26493696 & 4.38 & 43.46 & 7.4 & 12.3 & $<0.01$ & 19 & 0.92 \\
\hline 39 & Mrk10 & 26498304 & 3.12 & 43.79 & 6.2 & 20.3 & $<0.01$ & 22.7 & 0.83 \\
\hline 40 & IC 486 & 18971136 & 3.22 & 43.73 & 5.4 & 11.3 & 0.020 & 42 & 0.74 \\
\hline 41 & Phoenix & 25408256 & 5.34 & 43.34 & 35 & 18 & $<0.01$ & 202 & 0.78 \\
\hline 42 & 2MAS X0904 & 26495744 & 1.91 & 43.78 & 2.1 & 3.2 & 0.117 & 13 & $\ldots$ \\
\hline 43 & 2MAS X0911 & 26496256 & 1.81 & 43.47 & 2.9 & 6.4 & 0.070 & 25 & $\ldots$ \\
\hline 44 & MCG-01-24-012 & 18945024 & 4.58 & 43.60 & 6.2 & 10.5 & $<0.01$ & 53 & 0.53 \\
\hline 45 & $\mathrm{MCG}+04-22-042$ & 26491392 & 4.46 & 44.03 & 5.9 & 8.2 & $<0.01$ & 70 & 1.11 \\
\hline 46 & Mrk 110 & 14189824 & 6.15 & 44.25 & 3.0 & 4.1 & $<0.01$ & 44 & 1.17 \\
\hline 47 & Mrk 705 & 14203392 & 2.13 & 43.62 & 5.1 & 5.4 & 0.021 & 71 & 1.0 \\
\hline 48 & NGC 2992 & 26122240,4934144 & 4.82 & 42.80 & 88 & 101 & 0.085 & 130 & $\cdots$ \\
\hline 49 & MCG -05-23-016 & 26484992 & 20.77 & 43.52 & 18.1 & 27 & $<0.01$ & 337 & 0.69 \\
\hline 50 & NGC 3081 & 4851968,18509824 & 10.24 & 43.16 & 44 & 97 & $<0.01$ & 107 & 0.75 \\
\hline 51 & NGC 3079 & 3755520 & 3.44 & 42.02 & 29 & 14 & 0.41 & 864 & $\ldots$ \\
\hline 52 & ESO 374 G44 & 26497792 & 2.82 & 43.72 & $\cdots$ & 22 & $<0.01$ & 25.7 & 0.8 \\
\hline 53 & NGC 3227 & 4934656 & 14.13 & 42.67 & 64 & 65 & 0.101 & 296 & $\ldots$ \\
\hline 54 & NGC 3281 & 4852224 & 9.01 & 43.36 & $\cdots$ & $\cdots$ & $<0.01$ & 481 & 0.27 \\
\hline
\end{tabular}


Table 1-Continued

\begin{tabular}{|c|c|c|c|c|c|c|c|c|c|}
\hline No. & Name $^{\mathrm{a}}$ & $\mathrm{AOR}^{\mathrm{b}}$ & $\begin{array}{c}\operatorname{flux}(\mathrm{X})^{\mathrm{c}} \\
10^{-11}\end{array}$ & $\begin{array}{c}L(\mathrm{X})^{\mathrm{d}} \\
\log \operatorname{erg~s}^{-1}\end{array}$ & $\begin{array}{c}\mathrm{f}([\mathrm{NeIII}])^{\mathrm{e}} \\
10^{-21}\end{array}$ & $\begin{array}{c}\mathrm{f}([\mathrm{OIV}])^{\mathrm{f}} \\
10^{-21}\end{array}$ & $\begin{array}{c}\mathrm{EW}(6.2 \mu \mathrm{m})^{\mathrm{g}} \\
\mu \mathrm{m}\end{array}$ & $\begin{array}{c}f_{\nu}(7.8 \mu \mathrm{m})^{\mathrm{h}} \\
\mathrm{mJy}\end{array}$ & silicate $^{\mathrm{i}}$ \\
\hline 55 & Mrk 417 & 18946048 & 3.74 & 43.97 & 3.2 & 5.2 & $<0.01$ & 32 & 0.88 \\
\hline 56 & UGC 06527 & 26868992 & 2.68 & 43.68 & 8.7 & 10.7 & $<0.01$ & 52 & 0.93 \\
\hline 57 & NGC 3783 & 4852736,18510592 & 19.45 & 43.61 & 25 & 34 & $<0.01$ & 317 & 1.0 \\
\hline 58 & UGC 06728 & 26483712 & 2.95 & 42.44 & 1.3 & 3.3 & $<0.01$ & 20 & 1.11 \\
\hline 59 & 2MASX J11454045 & 26484736 & 6.38 & 44.20 & 6.1 & 24 & $<0.01$ & 40 & 1.0 \\
\hline 60 & NGC 3998 & 10512896 & 3.03 & 41.91 & $\ldots$ & $\ldots$ & 0.01 & 40 & 1.29 \\
\hline 61 & NGC 4051 & 14449152 & 4.34 & 41.71 & $\ldots$ & $\ldots$ & 0.044 & 281 & 0.94 \\
\hline 62 & Ark 347 & 26483456 & 3.85 & 43.64 & 12.2 & 28.5 & $<0.01$ & 50 & 1.0 \\
\hline 63 & NGC 4102 & 18941952 & 2.58 & 41.66 & 37 & $<26$ & 0.311 & 1080 & $\ldots$ \\
\hline 64 & NGC 4151 & 3754496 & 62.23 & 43.18 & 230 & 214 & $<0.01$ & 970 & 0.96 \\
\hline 65 & NGC 4235 & 22086144 & 2.40 & 42.54 & 6.3 & 3.7 & $<0.01$ & 23 & 1.0 \\
\hline 66 & Mrk 766 & 14448896 & 2.42 & 42.96 & $\ldots$ & $\ldots$ & 0.042 & 199 & 0.72 \\
\hline 67 & M106 & 4934912,18526208 & 2.80 & 41.14 & 12.0 & 6.3 & 0.024 & 79 & 1.13 \\
\hline 68 & Mrk 50 & 26496768 & 3.53 & 43.64 & $\cdots$ & $\cdots$ & 0.01 & 17 & 1.16 \\
\hline 69 & NGC 4395 & 17792256 & 3.12 & 40.89 & 2.5 & 4.4 & $<0.01$ & 3.7 & 1.0 \\
\hline 70 & NGC 4388 & 4852992,18510848 & 34.64 & 43.74 & 134 & 295 & 0.044 & 238 & 0.40 \\
\hline 71 & $3 \mathrm{C} 273$ & 19718656 & 38.35 & 46.42 & 3.2 & 7.8 & $<0.01$ & 276 & 1.0 \\
\hline 72 & NGC 4507 & 18511104,4853248 & 22.51 & 43.85 & 29 & 35 & $<0.01$ & 349 & 1.0 \\
\hline 73 & ESO 506 & 18941696 & 13.75 & 44.29 & 4.3 & 3.9 & $<0.01$ & 131 & 0.32 \\
\hline 74 & NGC 4593 & 4853504 & 9.79 & 43.25 & $\cdots$ & $\cdots$ & $<0.01$ & 204 & 1.04 \\
\hline 75 & NGC 4686 & 26492160 & 3.08 & 43.29 & 1.7 & 1.1 & $<0.01$ & 17 & 0.77 \\
\hline 76 & SBS 1301 & 26498048 & 4.02 & 43.92 & 0.36 & 0.28 & $<0.01$ & 10 & 1.21 \\
\hline 77 & NGC 4945 & 8768000,8769280 & 32.98 & 42.41 & 109 & $\ldots$ & 0.21 & 2940 & $\ldots$ \\
\hline 78 & ESO $323-077$ & 18942720 & 4.70 & 43.38 & 17.2 & 29 & 0.029 & 426 & 0.96 \\
\hline 79 & NGC 4992 & 26490880 & 6.16 & 43.95 & $<0.8$ & 1.1 & $<0.01$ & 65 & 0.34 \\
\hline 80 & MCG-03-34-064 & 20367615 & 3.15 & 43.29 & 121 & 106 & $<0.01$ & 410 & 0.74 \\
\hline 81 & Cen A & 8766464,26121984 & 92.62 & 42.83 & $\cdots$ & $\cdots$ & 0.01 & 891 & 0.39 \\
\hline
\end{tabular}


Table 1-Continued

\begin{tabular}{|c|c|c|c|c|c|c|c|c|c|}
\hline No. & $\mathrm{Name}^{\mathrm{a}}$ & $\mathrm{AOR}^{\mathrm{b}}$ & $\begin{array}{c}\text { flux }(\mathrm{X})^{\mathrm{c}} \\
10^{-11}\end{array}$ & $\begin{array}{c}L(\mathrm{X})^{\mathrm{d}} \\
\log \operatorname{erg~s}^{-1}\end{array}$ & $\begin{array}{c}\mathrm{f}([\mathrm{NeIII}])^{\mathrm{e}} \\
10^{-21}\end{array}$ & $\begin{array}{c}\mathrm{f}([\mathrm{OIV}])^{\mathrm{f}} \\
10^{-21}\end{array}$ & $\begin{array}{c}\mathrm{EW}(6.2 \mu \mathrm{m})^{\mathrm{g}} \\
\mu \mathrm{m}\end{array}$ & $\begin{array}{c}f_{\nu}(7.8 \mu \mathrm{m})^{\mathrm{h}} \\
\text { mJy }\end{array}$ & silicate $^{\mathrm{i}}$ \\
\hline 82 & MCG-06-30-015 & 4849920 & 7.82 & 43.02 & 8 & 18 & $<0.01$ & 125 & 0.92 \\
\hline 83 & NGC 5252 & 18946304 & 8.18 & 43.99 & 6.0 & 8.7 & $<0.01$ & 32 & 1.0 \\
\hline 84 & IC $4329 \mathrm{~A}$ & 18506496 & 33.08 & 44.28 & 63 & 111 & $<0.01$ & 616 & 0.93 \\
\hline 85 & UM 614 & 26497024 & 2.24 & 43.74 & 2.1 & 5.7 & $<0.01$ & 20 & 1.0 \\
\hline 86 & Mrk 279 & 7616512 & 5.30 & 44.05 & 8.7 & 13.6 & 0.008 & 111 & 1.0 \\
\hline 87 & Circinus & 9074176 & 27.48 & 42.10 & $\ldots$ & .. & 0.033 & 7850 & 0.28 \\
\hline 88 & NGC 5506 & 18512896,4855040 & 25.64 & 43.34 & 157 & 240 & 0.007 & 1030 & 0.43 \\
\hline 89 & NGC 5548 & 18513152,4855296 & 8.08 & 43.73 & 9.5 & 9.7 & 0.021 & 110 & 0.97 \\
\hline 90 & ESO 511 & 26491136 & 4.71 & 43.73 & 1.8 & 0.8 & $<0.01$ & 40 & 1.38 \\
\hline 91 & NGC 5728 & 18945536 & 10.54 & 43.31 & 58 & 109 & 0.12 & 115 & $\cdots$ \\
\hline 92 & Mrk 841 & 3761664 & 2.93 & 43.96 & 9.7 & 20 & $<0.01$ & 92 & 0.94 \\
\hline 93 & Mrk 1392 & 15079168 & 1.99 & 43.78 & 8.9 & 17.1 & $<0.01$ & 34 & 1.0 \\
\hline 94 & NGC 5899 & 22090240 & 2.15 & 42.54 & 18 & 25 & $<0.01$ & 24 & 0.27 \\
\hline 95 & NGC 6240 & 4985600 & 7.30 & 44.00 & 66 & 43 & 0.28 & 463 & $\ldots$ \\
\hline 96 & NGC 6300 & 22091264 & 10.35 & 42.50 & 15 & 29 & 0.026 & 319 & 0.26 \\
\hline 97 & HB89(1821) & 4676096 & 1.74 & 45.69 & 11.0 & 23 & $<0.01$ & 127 & 1.0 \\
\hline 98 & 3C 380 & 4581888 & 2.56 & 46.73 & 0.48 & $\cdots$ & $\cdots$ & 15 & 1.11 \\
\hline 99 & 3C 382 & 11306496 & 8.42 & 44.83 & 1.6 & 1.7 & $<0.01$ & 83 & 1.11 \\
\hline 100 & Fairall49 & 18507520 & 2.93 & 43.43 & 41 & 37 & 0.021 & 387 & 0.72 \\
\hline 101 & ESO 103-G035 & 18505728,4847872 & 11.14 & 43.64 & 31 & 31 & $<0.01$ & 176 & 0.39 \\
\hline 102 & 3C 390.3 & 4673024 & 10.97 & 44.92 & 2.8 & 1.9 & $<0.01$ & 62 & 1.0 \\
\hline 103 & Fairall51 & 26489088 & 4.57 & 43.31 & 15.9 & 22.2 & 0.025 & 239 & 1.0 \\
\hline 104 & ESO 141 G55 & 26489856 & 5.27 & 44.20 & 4.3 & 6.9 & $<0.01$ & 113 & 1.18 \\
\hline 105 & NGC 6814 & 22091776 & 7.82 & 42.67 & 15 & 18 & $<0.01$ & 69 & 1.0 \\
\hline 106 & CygnusA & 4673280 & 12.23 & 44.96 & 42 & 63 & $<0.01$ & 54 & 0.43 \\
\hline 107 & MCG 0448 & 20349184 & 8.77 & 43.58 & 22.5 & 17.6 & 0.48 & 347 & . \\
\hline 108 & Mrk 509 & 4850432,18508288 & 9.44 & 44.41 & 13.7 & 26 & 0.019 & 191 & 0.97 \\
\hline
\end{tabular}


Table 1-Continued

\begin{tabular}{|c|c|c|c|c|c|c|c|c|c|}
\hline No. & Name $^{\mathrm{a}}$ & $\mathrm{AOR}^{\mathrm{b}}$ & $\begin{array}{c}\operatorname{flux}(\mathrm{X})^{\mathrm{c}} \\
10^{-11}\end{array}$ & $\begin{array}{c}L(\mathrm{X})^{\mathrm{d}} \\
\log \operatorname{erg~s}^{-1}\end{array}$ & $\begin{array}{c}\mathrm{f}([\mathrm{NeIII}])^{\mathrm{e}} \\
\quad 10^{-21}\end{array}$ & $\begin{array}{c}\mathrm{f}([\mathrm{OIV}])^{\mathrm{f}} \\
10^{-21}\end{array}$ & $\begin{array}{c}\mathrm{EW}(6.2 \mu \mathrm{m})^{\mathrm{g}} \\
\mu \mathrm{m}\end{array}$ & $\begin{array}{c}f_{\nu}(7.8 \mu \mathrm{m})^{\mathrm{h}} \\
\mathrm{mJy}\end{array}$ & silicate $^{\mathrm{i}}$ \\
\hline 109 & IC 5063 & 18506752 & 8.59 & 43.39 & 73 & 91 & $<0.01$ & 454 & 0.69 \\
\hline 110 & 3C 433 & 11307264 & 1.74 & 44.66 & 2.7 & 7.0 & $<0.01$ & 47 & 0.42 \\
\hline 111 & Mrk 520 & 26490112 & 3.58 & 43.76 & 31 & 50 & 0.10 & 147 & $\cdots$ \\
\hline 112 & NGC 7172 & 4856064,18513920 & 18.11 & 43.48 & 20 & 43 & 0.036 & 275 & 0.13 \\
\hline 113 & NGC 7213 & 18514176,4856320 & 5.75 & 42.64 & 15 & 2.2 & $<0.01$ & 102 & 1.44 \\
\hline 114 & NGC 7314 & 4856576,18514432 & 4.63 & 42.37 & 24 & 50 & $<0.01$ & 45 & 0.57 \\
\hline 115 & Mrk 915 & 26495488 & 4.99 & 43.82 & 18.8 & 39.2 & $<0.01$ & 33 & 0.91 \\
\hline 116 & $3 \mathrm{C} 452$ & 11301632 & 3.78 & 44.79 & 1.36 & 0.54 & $<0.01$ & 12.8 & 0.83 \\
\hline 117 & UGC 12282 & 26492672 & 2.49 & 43.21 & 2.2 & 3.0 & $<0.01$ & 22 & 0.74 \\
\hline 118 & NGC 7469 & 3755008 & 6.66 & 43.60 & 38 & 45 & 0.137 & 764 & $\cdots$ \\
\hline 119 & Mrk 926 & 4856832 & 10.17 & 44.72 & 5.3 & 10.0 & $<0.01$ & 59 & 0.96 \\
\hline 120 & NGC 7582 & 26121728,3855616 & 7.92 & 42.68 & 118 & 168 & 0.285 & 134 & $\cdots$ \\
\hline 121 & NGC 7603 & 10870784 & 4.70 & 43.97 & 4.0 & 6.2 & 0.018 & 227 & 1.13 \\
\hline 122 & LCRS B232242.2 & 26497280 & 2.41 & 43.86 & $\cdots$ & $\cdots$ & 0.073 & 27 & $\cdots$ \\
\hline 123 & NGC 7682 & 26494208 & 2.27 & 43.18 & 9.3 & 12.1 & 0.08 & 7 & 1.0 \\
\hline 124 & NGC 7679 & 20350720 & 2.33 & 43.19 & 30 & 27.5 & 0.379 & 261 & $\cdots$ \\
\hline 125 & UGC 12741 & 26494720 & 4.00 & 43.44 & 1.3 & 2.2 & 0.08 & 14 & 0.40 \\
\hline
\end{tabular}

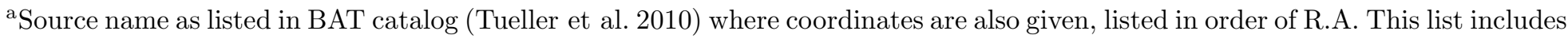
all objects listed with "Sy" or "quasar" classification in BAT catalog ("Type" in their Table 5) which also have low resolution IRS spectra; objects listed as "blazar" are not included.

${ }^{\mathrm{b}}$ AOR number for Spitzer IRS spectra available in CASSIS.

${ }^{\mathrm{c}}$ Hard X-ray flux (14-195 keV) from Tueller et al. (2010) in units of $10^{-11} \mathrm{erg} \mathrm{s}^{-1} \mathrm{~cm}^{-2}$.

${ }^{\mathrm{d}}$ Hard X-ray luminosity from Tueller et al. (2010) in $\log \mathrm{erg} \mathrm{s}^{-1}$. 
eTotal flux of [NeIII] $15.55 \mu \mathrm{m}$ emission line fit with single gaussian in low resolution CASSIS spectra in units of $10^{-21} \mathrm{~W} \mathrm{~cm}^{-2}$.

${ }^{\mathrm{f}}$ Total flux of [OIV] $25.89 \mu \mathrm{m}$ emission line fit with single gaussian in low resolution CASSIS spectra in units of $10^{-21} \mathrm{~W} \mathrm{~cm}^{-2}$.

${ }^{\mathrm{g}}$ Rest frame equivalent width of $6.2 \mu \mathrm{m}$ PAH feature fit with single gaussian on a linear continuum within rest wavelength range $5.5 \mu \mathrm{m}$ to $6.9 \mu \mathrm{m}$.

${ }^{\mathrm{h}}$ Flux density $f_{\nu}(7.8 \mu \mathrm{m})$ from IRS low resolution spectra at observed wavelength corresponding to rest wavelength $7.8 \mu \mathrm{m}$.

${ }^{i}$ Strength of silicate feature defined as $f_{\nu}(10 \mu \mathrm{m}$ observed $) / f_{\nu}(10 \mu \mathrm{m}$ continuum $)$, for $f_{\nu}(10 \mu \mathrm{m}$ continuum $)$ extrapolated linearly between $f_{\nu}(7.8 \mu \mathrm{m})$ and $f_{\nu}(13 \mu \mathrm{m})$. Values $>1$ correspond to silicate emission and values $<1$ to silicate absorption. No silicate strength is given for sources with $\mathrm{EW}(6.2 \mu \mathrm{m})>0.05 \mu \mathrm{m}$ because of possible contamination of the extrapolated continuum at 7.8 $\mu \mathrm{m}$ by PAH $7.7 \mu \mathrm{m}$ emission. 
As initially demonstrated by Genzel et al. (1998), the strength of the PAH features measures the relative starburst/AGN components because PAH emission is associated with the starburst component. In various previous summaries of IRS spectra using the same measures we use for the BAT AGN in Table 1, we have illustrated for large samples how the equivalent width of the $6.2 \mu \mathrm{m}$ feature quantitatively correlates with the starburst/AGN classifications (Sargsyan et al. 2011, 2012 and references therein). The summary result we adopted from these previous studies is that sources having $\mathrm{EW}(6.2 \mu \mathrm{m})<0.1 \mu \mathrm{m}$ have the majority of luminosity at $7.8 \mu \mathrm{m}$ arising from an AGN, and our starburst/AGN classification from IRS spectra is based only on this EW criterion, which is also applied to luminous DOGs at high redshift (Weedman and Houck 2009). The BAT sample in Table 1 confirms this generalized PAH classification criterion. Of the 125 AGN with IRS spectra, only 12 have $\operatorname{EW}(6.2 \mu \mathrm{m})>0.1 \mu \mathrm{m}$. These exceptions are explainable as sources for which the AGN continuum luminosity is weak in the infrared compared to the luminosity from a circumnuclear starburst.

Figure 2 shows the silicate absorption or emission strength from CASSIS spectra compared to optical AGN classification for Swift BAT AGN in Table 1, using the optical classifications given in Tueller et al. (2010). (As explained in section 2.1, no silicate strength is given for the 20 sources with $\mathrm{EW}(6.2 \mu \mathrm{m})>0.05 \mu \mathrm{m}$ to avoid possible contamination of the $7.8 \mu \mathrm{m}$ continuum by $7.7 \mu \mathrm{m}$ PAH emission.) The overall consistency between infrared and optical classifications is clearly demonstrated in Figure 2. As expected, the sources with silicate absorption are predominately type 2 and those with silicate emission predominately type 1 .

There are ambiguous sources and a wide range of silicate strength at a given optical classification. This is not surprising given that the broad optical emission lines that classify type 1 can sometimes be seen via scattering from intervening dust. In fact, the observation of this scattering in polarized light led to the initial "unified theory" (Antonucci and Miller 1985). An important example of an ambiguous classification is the well studied ULIRG Markarian 231. This object has silicate absorption of strength 0.5 in the infrared and extensive multiwavelength evidence of heavy absorption (Boksenberg et al. 1977; Gallagher et al. 2005) but is optically a type 1 AGN. Such ambiguities are a primary motive for classification based only on the silicate strength, which provides a quantitative measure of the presence or absence of intervening dust between observer and AGN.

For example, the dust covering factor can be estimated by the fraction of AGN seen with silicate absorption. Within the BAT AGN sample, the fraction of sources with measured absorption (silicate strength less than 1 among those with measured strength in Table 1) is $58 / 105$, or $55 \%$. This implies that of all AGN, 55\% are observed through dust clouds. This 
could arise from various configurations. A uniform dusty torus might cover $55 \%$ of the solid angle around the AGN, or individual clouds in a spherical configuration could cover $55 \%$. For our purposes, the numerical value of the covering factor is the important result because that is a measure of the fraction of $L_{b o l}$ for AGN which is absorbed and reradiated as $L_{I R}$.

\subsection{Infrared Luminosities for the BAT AGN Sample}

The primary result we desire from studying IRS spectra of the BAT sample is to calibrate how well the mid-infrared continuum luminosity $\nu L_{\nu}(7.8 \mu \mathrm{m})$ is a measure of total AGN luminosity for AGN of all classifications, because we use this mid-infrared luminosity parameter for the high redshift DOGs and other dusty quasars. We also will compare to the emission line luminosity of the mid-infrared [OIV] $25.89 \mu \mathrm{m}$ emission line, because of previous conclusions that this line is an indicator of intrinsic AGN luminosity (Rigby et al. 2009; Weaver et al. 2010).

The comparisons between infrared emission lines and BAT X-ray properties by Weaver et al. (2010) used 79 sources with IRS high resolution spectra. This sample is enlarged if we use the 125 sources in Table 1 with low resolution spectra. The [OIV] line is strong enough to measure in most of these low resolution spectra, but the lower contrast of emission lines in low resolution spectra means the measurement of line fluxes is more uncertain because of continuum noise. To use these measurements, we need an estimate of the line flux uncertainty. This is estimated by comparing the CASSIS low resolution measures with the high resolution measures for the objects in common. To extend these comparisons, we also use the [NeIII] $15.55 \mu \mathrm{m}$ line, which is of comparable strength to [OIV].

The emission line measurements from IRS high resolution and low resolution spectra are independent. The high resolution fitting and measurements in Weaver et al. (2010) are based on calibrations for the high resolution spectra provided by data products from the Spitzer Science Center. The CASSIS low resolution results derive from independent calibration in the CASSIS process (Lebouteiller et al. 2011) and from gaussian line fits done with the SMART analyis package (Higdon et al. 2004; Lebouteiller et al. 2010).

Comparisons of low resolution and high resolution line fluxes are shown in Figure 3. The median and dispersion $\log [$ flux(high) $/$ flux(low) $]=-0.02 \pm 0.10$. The small systematic offset can be caused by small sample size, by differences in line fitting techniques, or by calibration differences; the total systematic difference of $\sim 5 \%$ is an upper limit to calibration differences. The dispersion in the results gives the random measurement uncertainty of $\sim 25 \%$. We cannot determine from Figure 3 how much of the random uncertainty is attributed to the 


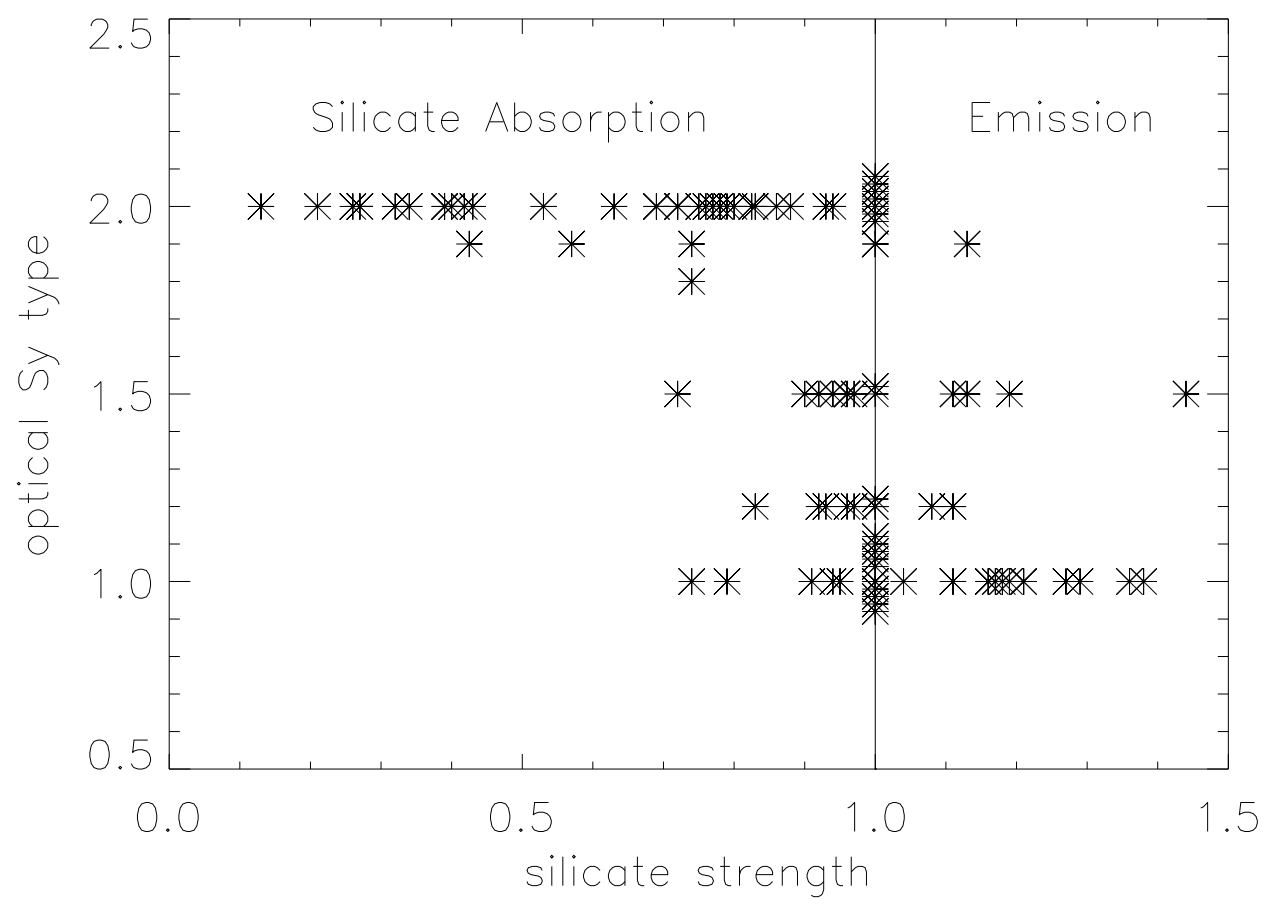

Fig. 2. - Silicate absorption or emission compared to optical AGN classification for all Swift BAT AGN having Spitzer IRS spectra and optical classifications in Tueller et al. (2010). Strength of the silicate feature is defined in Figure 1 and listed in Table 1. Values $>1$ correspond to silicate emission and values $<1$ to silicate absorption. Sources having optical classes 1.0 or 2.0 and silicate strength 1.0 are artificially displaced slightly in optical class to avoid overlapping symbols. 


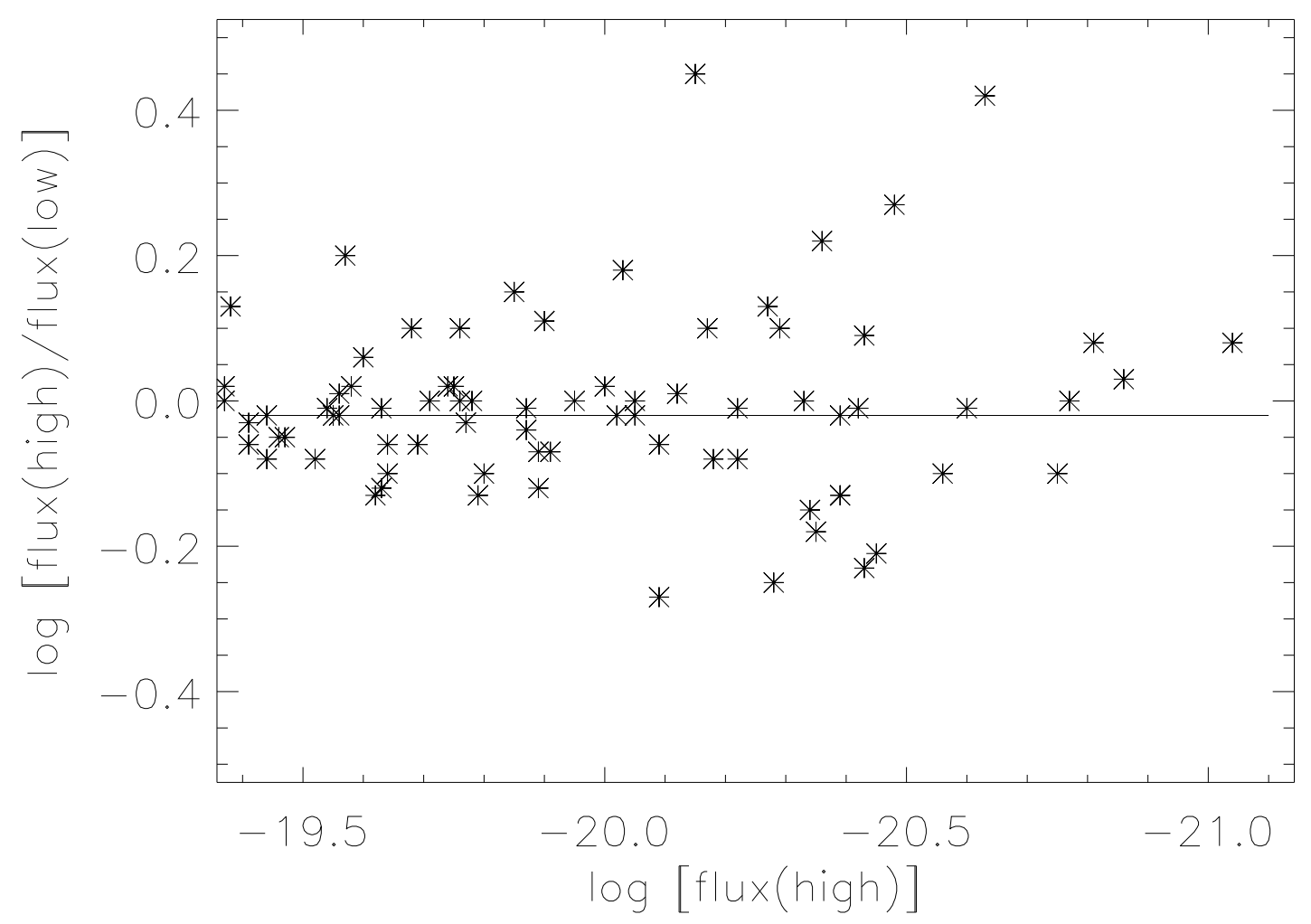

Fig. 3.- Comparison of emission line fluxes measured in IRS low resolution CASSIS spectra of the BAT AGN compared to high resolution measures from Weaver et al. (2011) for [NeIII] and [OIV]. Units of line flux are $\mathrm{W} \mathrm{cm}{ }^{-2}$. 


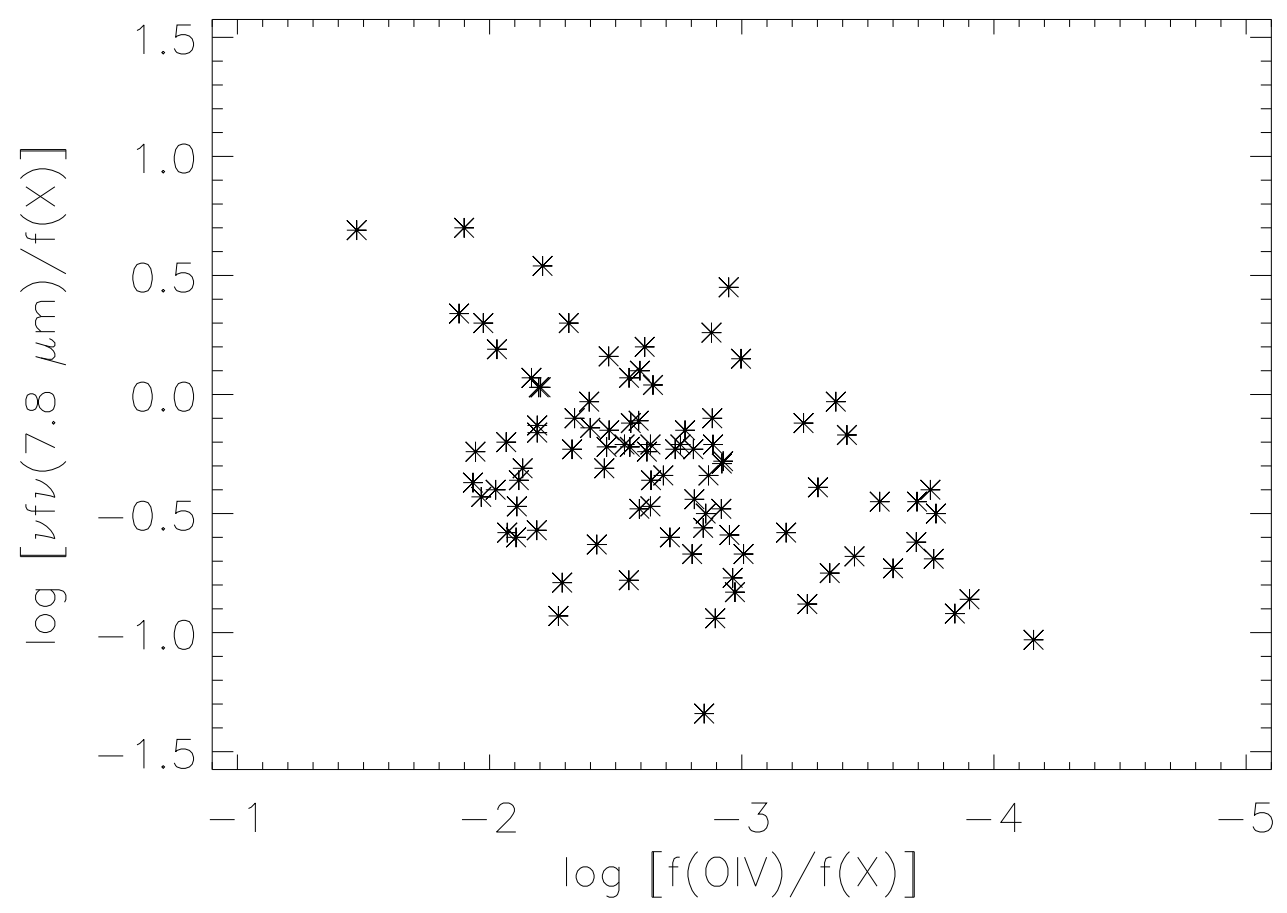

Fig. 4.- Ratio of [OIV] $25.89 \mu \mathrm{m}$ emission line flux to hard X-ray flux for BAT AGN, compared to ratio of mid-infrared flux to hard X-ray flux. Medians and dispersions are $\log \left[\nu f_{\nu}(7.8 \mu \mathrm{m}) / f(\mathrm{X})\right]=-0.31 \pm 0.35$ and $\log [f([\mathrm{OIV}] 25.89 \mu \mathrm{m}) / f(\mathrm{X})]=-2.64 \pm 0.6$. The smaller dispersion in comparing infrared with hard X-ray indicates that observed dust luminosity is a better measure of accretion luminosity than is the forbidden line luminosity. 


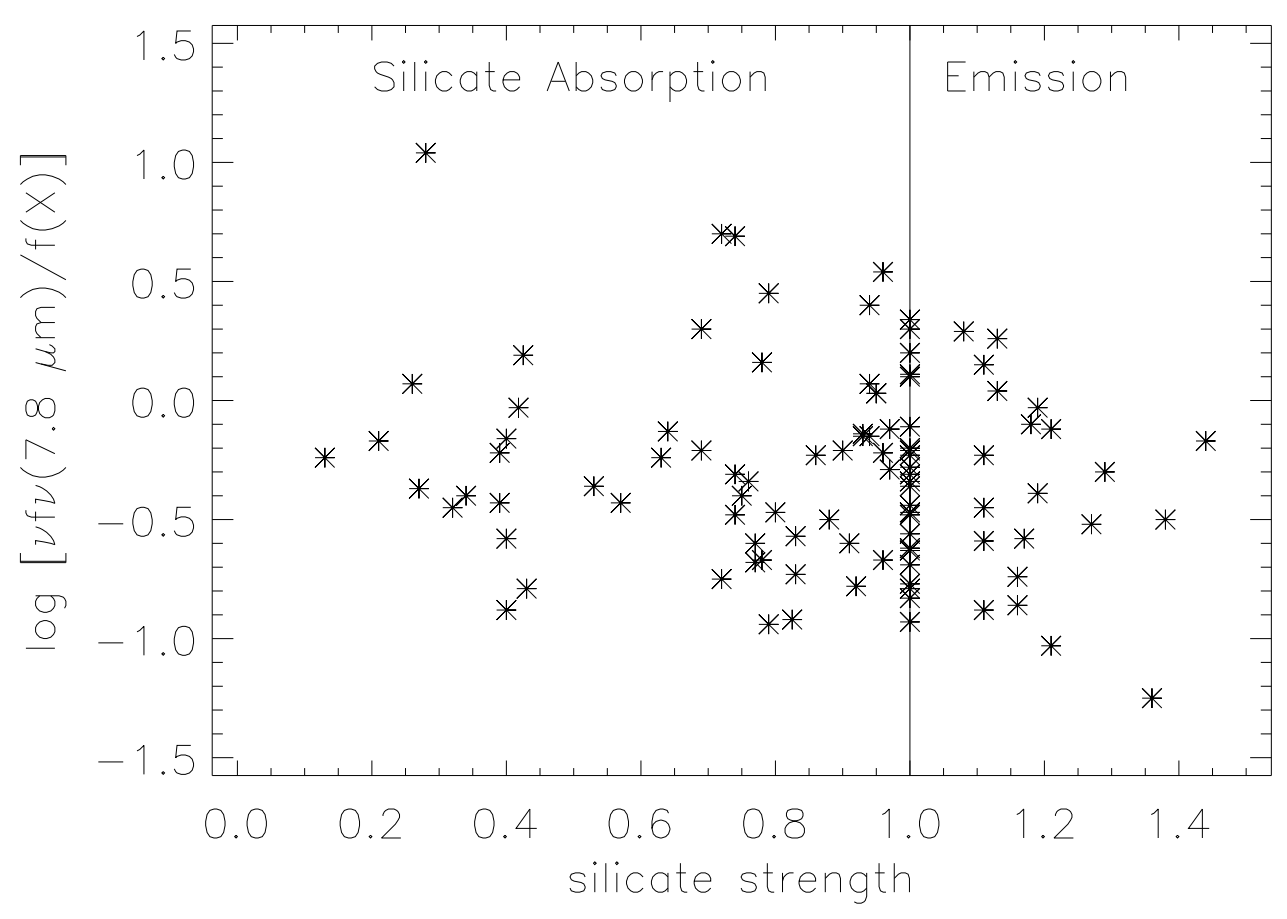

Fig. 5.- Ratio of mid-infrared flux to hard X-ray flux for BAT AGN, compared to silicate absorption or emission strength defined in Figure 1 and listed in Table 1 . Values $>1$ correspond to silicate emission and values $<1$ to silicate absorption. 
low resolution lines and how much to the high resolution, but we assume this dispersion to be a measure of the uncertainty only in low resolution fluxes because low resolution lines are more affected by localized continuum noise. Therefore, the dex \pm 0.10 dispersion of the ratio is taken as the uncertainty for the [OIV] line fluxes when compared to X-ray fluxes in Figure 4.

Three measures of AGN flux given in Table 1 are compared in Figure 4: 1. Hard X-ray flux, $f(\mathrm{X})$, from the BAT results, 2. [OIV] mid-infrared emission line flux, and 3. Mid-infrared continuum flux $\nu f_{\nu}(7.8 \mu \mathrm{m})$. In these comparisons, flux ratios or luminosity ratios are equivalent when luminosities are compared at the same rest frame wavelengths as the observed fluxes, so we can discuss correlations using either flux ratios or luminosity ratios. The $20 \mathrm{BAT}$ AGN in Table 1 with $\operatorname{EW}(6.2 \mu \mathrm{m} \mathrm{PAH})>0.05 \mu \mathrm{m}$ are not used in these comparisons to avoid possible contamination of the continuum $f_{\nu}(7.8 \mu \mathrm{m})$ by the 7.7 $\mu \mathrm{m}$ PAH feature. Medians and dispersions are $\log \left[\nu f_{\nu}(7.8 \mu \mathrm{m}) / f(\mathrm{X})\right]=-0.31 \pm 0.35$ and $\log$ $[f([\mathrm{OIV}] 25.89 \mu \mathrm{m}) / f(\mathrm{X})]=-2.64 \pm 0.6$. These dispersions quantitatively demonstrate that the $\nu f_{\nu}(7.8 \mu \mathrm{m})$ scales more closely with $f(\mathrm{X})$ than does [OIV] emission, and the dispersion compared to $f(\mathrm{X})$ provides our most unbiased calibration yet available of the precision with which the mid-infrared continuum tracks the primary luminosity from the AGN.

The measured dispersion of \pm 0.35 in dex means that the mid-infrared flux (or luminosity) predicts the hard X-ray flux (or luminosity) to a one sigma precision of about a factor of 2. This is a cosmic dispersion illustrating differences from source to source in the various physical characteristics that affect this ratio. Differences can arise from many effects including differences in covering factors and temperatures for the absorbing dust, and differences in the spectral energy distribution of the primary luminosity such that the luminosity at wavelengths absorbed by the dust differs in proportion to the hard X-ray luminosity. Because of the many factors that can enter the ratio $\nu f_{\nu}(7.8 \mu \mathrm{m}) / f(\mathrm{X})$ and the wide wavelength difference between these two observational measures, the resulting correlation illustrates (to us) surprising consistency among AGN.

The next question is whether the mid-infrared continuum luminosity represents the intrinsic luminosity regardless of AGN orientation and any extinction imposed by the accident of the observer's line of sight. This is tested in Figure 5, by considering the ratio of midinfrared flux (or luminosity) to X-ray flux (or luminosity) for BAT AGN when compared to the strength of silicate absorption or emission. The silicate strength is a measure of whether the AGN is observed through dust, as discussed in section 2.1. This Figure shows no trend for the ratio $\log \left[\nu f_{\nu}(7.8 \mu \mathrm{m}) / f(\mathrm{X})\right]$ to depend on silicate strength; the median ratio for emission sources (strength $\geq 1$ ) is -0.35 and for absorption sources (strength $<1$ ) is -0.3 . This result implies the important conclusion that the mid-infrared luminosity can be used 
equally well as a measure of intrinsic AGN luminosity regardless of AGN orientation and the resulting classification.

\section{Infrared Luminosities, Bolometric Luminosities, and Black Hole Masses}

The preceding sections establish the reliability of the luminosity measure $\nu L_{\nu}(7.8 \mu \mathrm{m})$ for AGN of any classification, so that AGN (or quasars) can be uniformly compared in luminosity regardless of the extinction effects that may seriously affect rest frame optical and ultraviolet measurements. In this section, we compare this measure of infrared luminosity to other measures of bolometric luminosity for a local AGN sample and for the most infraredluminous quasars now known. We also compare to the most fundamental physical parameter for AGN, which is the mass of the accreting supermassive black holes. These comparisons illustrate the differences and dispersions between very different methods of measuring luminosity.

Long term variability studies of AGN combined with observed velocity dispersions in the broad line region (BLR) led to the "reverberation mapping" technique for measuring the virial masses of black holes in type 1 AGN (Peterson et al. 2004). The primary set of reverberation mapped AGN contains 35 sources with black hole masses, of which 32 have CASSIS spectra. These are summarized in Table 2 and are the local AGN sample for which we compare $\nu L_{\nu}(7.8 \mu \mathrm{m})$ to black hole masses and $L_{b o l}$.

Because reverberation mapping works only if the BLR is observable, these local AGN are of necessity type 1 . To extend these comparisons to the most luminous type 1 sources known, we also summarize the most infrared-luminous type 1 quasars. These arise from the wide area SDSS survey combined with infrared photometry from WISE. Finally, the most luminous type 1 quasars are compared to the infrared luminosities of the heavily obscured quasars among the DOGs discovered spectroscopically by Spitzer and among those estimated photometrically from WISE. 
Table 2. Observed Properties of AGN with Black Hole Masses

\begin{tabular}{|c|c|c|c|c|c|c|c|c|c|}
\hline No. & $\mathrm{Name}^{\mathrm{a}}$ & $\mathrm{AOR}^{\mathrm{b}}$ & $\mathrm{z}$ & $\begin{array}{c}\mathrm{EW}(6.2 \mu \mathrm{m})^{\mathrm{c}} \\
\mu \mathrm{m}\end{array}$ & $\begin{array}{c}f_{\nu}(7.8 \mu \mathrm{m})^{\mathrm{d}} \\
\mathrm{mJy}\end{array}$ & $\begin{array}{c}\nu L_{\nu}(7.8 \mu \mathrm{m})^{\mathrm{e}} \\
\log \mathrm{erg} \mathrm{s}^{-1}\end{array}$ & $\begin{array}{c}L_{I R}^{\mathrm{f}} \\
\log \mathrm{L}_{\odot} \\
\end{array}$ & $\begin{array}{r}L_{b o l}{ }^{g} \\
\log \mathrm{L}_{\odot}\end{array}$ & $\begin{array}{c}\mathrm{BHM}^{\mathrm{h}} \\
\log \mathrm{M}_{\odot}\end{array}$ \\
\hline 1 & Mrk 335 & 14448128 & 0.0258 & $<0.01$ & 128.0 & 43.82 & 10.74 & 11.15 & 7.15 \\
\hline 2 & PG $0026+129$ & 10449408,14188800 & 0.1420 & $<0.01$ & 14.9 & 44.39 & 11.31 & 12.33 & 8.59 \\
\hline 3 & PG $0052+251$ & 4675072 & 0.1550 & $<0.01$ & 27.5 & 44.73 & 11.65 & 12.15 & 8.57 \\
\hline 4 & Fairall9 & 18505984,28720896 & 0.0470 & $<0.01$ & 197.0 & 44.52 & 11.44 & 11.32 & 8.41 \\
\hline 5 & Mrk 590 & 4850688,18508544 & 0.0264 & $<0.01$ & 39.0 & 43.32 & 10.24 & 10.82 & 7.68 \\
\hline 6 & 3C 120 & 18505216,4847360 & 0.0330 & $<0.01$ & 138.0 & 44.06 & 10.98 & 11.47 & 7.74 \\
\hline 7 & Ark 120 & 18941440 & 0.0323 & $<0.01$ & 177.0 & 44.15 & 11.07 & 11.24 & 8.18 \\
\hline 8 & Mrk 79 & $\cdots$ & $\cdots$ & $\ldots$ & $\cdots$ & $\cdots$ & $\cdots$ & 11.03 & 7.72 \\
\hline 9 & PG $0804+761$ & 9074944 & 0.1000 & $<0.01$ & 100.0 & 44.90 & 11.82 & 12.26 & 8.84 \\
\hline 10 & PG $0844+349$ & 10449664,14189568 & 0.0640 & $<0.01$ & 32.0 & 44.01 & 10.93 & 11.56 & 7.97 \\
\hline 11 & Mrk 110 & 14189824 & 0.0353 & $<0.01$ & 44.0 & 43.62 & 10.54 & 11.01 & 7.40 \\
\hline 12 & PG $0953+414$ & 4675328 & 0.2341 & $<0.01$ & 22.0 & 45.00 & 11.92 & 12.53 & 8.44 \\
\hline 13 & NGC 3227 & 4934656 & 0.0039 & 0.10 & 296.0 & 42.74 & 9.66 & 10.00 & 7.63 \\
\hline 14 & NGC 3516 & $\cdots$ & $\cdots$ & $\cdots$ & $\cdots$ & $\cdots$ & $\cdots$ & 10.00 & 7.63 \\
\hline 15 & NGC 3783 & 4852736,18510592 & 0.0097 & $<0.01$ & 317.0 & 43.45 & 10.37 & 10.39 & 7.47 \\
\hline 16 & NGC 4051 & 14449152 & 0.0023 & 0.04 & 281.0 & 42.30 & 9.22 & 9.26 & 6.28 \\
\hline 17 & NGC 4151 & 3754496 & 0.0033 & $<0.01$ & 970.0 & 43.10 & 10.02 & 9.30 & 7.12 \\
\hline 18 & PG $1211+143$ & 3760896 & 0.0809 & $<0.01$ & 94.0 & 44.68 & 11.60 & 12.08 & 8.16 \\
\hline 19 & PG $1226+023(3 \mathrm{C} 273)$ & 19718656 & 0.1583 & $<0.01$ & 276.0 & 45.75 & 12.67 & 13.31 & 8.95 \\
\hline 20 & PG $1229+204$ & 10455296,14194944 & 0.0630 & $<0.01$ & 30.0 & 43.97 & 10.89 & 11.03 & 7.86 \\
\hline 21 & NGC 4593 & 4853504 & 0.0090 & $<0.01$ & 204.0 & 43.20 & 10.12 & 10.23 & 6.73 \\
\hline 22 & PG $1307+085$ & 4735488 & 0.1550 & $<0.01$ & 22.0 & 44.63 & 11.55 & 12.20 & 8.64 \\
\hline 23 & IC $4329 \mathrm{~A}$ & 18506496 & 0.0161 & $<0.01$ & 616.0 & 44.08 & 11.00 & 10.26 & 7.00 \\
\hline 24 & Mrk 279 & 7616512 & 0.0305 & $<0.01$ & 111.0 & 43.89 & 10.81 & 11.04 & 7.54 \\
\hline 25 & PG $1411+442$ & 10451456,10949888 & 0.0896 & $<0.01$ & 75.0 & 44.68 & 11.60 & 11.90 & 8.65 \\
\hline 26 & NGC 5548 & 18513152,4855296 & 0.0172 & 0.02 & 110.0 & 43.39 & 10.31 & 10.67 & 7.83 \\
\hline 27 & PG $1426+015$ & 10451712,14198272 & 0.0865 & $<0.01$ & 71.0 & 44.62 & 11.54 & 11.97 & 9.11 \\
\hline
\end{tabular}


Table 2-Continued

\begin{tabular}{cccccccccc}
\hline \hline No. & \multirow{2}{*}{ Name $^{\mathrm{a}}$} & $\mathrm{AOR}^{\mathrm{b}}$ & $\mathrm{z}$ & $\begin{array}{c}\mathrm{EW}(6.2 \mu \mathrm{m})^{\mathrm{c}} \\
\mu \mathrm{m}\end{array}$ & $\begin{array}{c}f_{\nu}(7.8 \mu \mathrm{m})^{\mathrm{d}} \\
\mathrm{mJy}\end{array}$ & $\begin{array}{c}\nu L_{\nu}(7.8 \mu \mathrm{m})^{\mathrm{e}} \\
\log \mathrm{erg} \mathrm{s}^{-1}\end{array}$ & $\begin{array}{c}L_{I R} \mathrm{f} \\
\log \mathrm{L}_{\odot}\end{array}$ & $\begin{array}{c}L_{b o l} \mathrm{~g} \\
\log \mathrm{L}_{\odot}\end{array}$ & $\begin{array}{c}\mathrm{BHM}^{\mathrm{h}} \\
\log \mathrm{M}_{\odot}\end{array}$ \\
\hline 28 & Mrk 817 & $\ldots$ & $\ldots$ & $\ldots$ & $\ldots$ & $\ldots$ & $\ldots$ & 11.04 & 7.69 \\
29 & PG 1613+658 & 10452480,14201344 & 0.1290 & $<0.01$ & 74.0 & 45.00 & 11.92 & 12.11 & 8.45 \\
30 & PG 1617+175 & 10452736,14201600 & 0.1124 & $<0.01$ & 28.0 & 44.45 & 11.37 & 11.74 & 8.77 \\
31 & PG 1700+518 & 4675840 & 0.2920 & $<0.01$ & 69.0 & 45.70 & 12.62 & 12.94 & 8.89 \\
32 & 3C 390.3 & 4673024 & 0.0561 & $<0.01$ & 62.0 & 44.18 & 11.10 & 11.02 & 8.46 \\
33 & Mrk 509 & 4850432,18508288 & 0.0344 & 0.02 & 191.0 & 44.24 & 11.16 & 11.54 & 8.16 \\
34 & PG 2130+099 & 3761408 & 0.0630 & $<0.01$ & 117.0 & 44.56 & 11.48 & 11.78 & 8.66 \\
35 & NGC 7469 & 3755008 & 0.0163 & 0.14 & 764.0 & 44.19 & 11.11 & 10.68 & 7.09 \\
\hline
\end{tabular}

a Source name as listed in Peterson et al. (2004), listed in order of R.A. Many of these sources are also in the BAT sample of Table 1.

${ }^{\mathrm{b}}$ AOR number for Spitzer IRS spectra available in CASSIS.

${ }^{\mathrm{c}}$ Rest frame equivalent width of $6.2 \mu \mathrm{m}$ PAH feature fit with single gaussian on a linear continuum within rest wavelength range $5.5 \mu \mathrm{m}$ to $6.9 \mu \mathrm{m}$.

${ }^{\mathrm{d}}$ Flux density $f_{\nu}(7.8 \mu \mathrm{m})$ from IRS low resolution spectra at observed wavelength corresponding to rest wavelength $7.8 \mu \mathrm{m}$.

${ }^{\mathrm{e}}$ Rest frame luminosity $\nu L_{\nu}(7.8 \mu \mathrm{m})$ in $\operatorname{erg~s}^{-1}$ determined as $\nu L_{\nu}(7.8 \mu \mathrm{m})=4 \pi \mathrm{D}_{L}{ }^{2}[\nu /(1+\mathrm{z})] f_{\nu}(7.8 \mu \mathrm{m})$, for $\nu$ corresponding to $7.8 \mu \mathrm{m}$, taking luminosity distances from Wright (2006): http://www.astro.ucla.edu/ wright/CosmoCalc.html, for $\mathrm{H}_{0}=74$ $\mathrm{km} \mathrm{s}^{-1} \mathrm{Mpc}^{-1}, \Omega_{M}=0.27$ and $\Omega_{\Lambda}=0.73$.

${ }^{\mathrm{f}}$ Total infrared luminosity $L_{I R}$ determined using calibration $\log \left[L_{I R} / \nu L_{\nu}(7.8 \mu \mathrm{m})\right]=0.51$ from Sargsvan et al. (2011) (Log $\left.\left[\nu L_{\nu}\left(\mathrm{L}_{\odot}\right)\right]=\log \left[\nu L_{\nu}\left(\mathrm{erg} \mathrm{s}^{-1}\right)\right]-33.59.\right)$

${ }^{g}$ Optically-determined bolometric luminosity $L_{b o l}$ from $\lambda L_{\lambda}(0.51 \mu \mathrm{m})$ of the AGN component in Bentz et al. (2009), assuming a scaling of $L_{b o l}=9.26 \lambda L_{\lambda}(0.51 \mu \mathrm{m})$ from Richards et al. (2006). 
${ }^{\mathrm{h}}$ Virial black hole mass from Peterson et al. (2004). 
The sample of SDSS/WISE quasars was selected using the following steps. Starting with the 105783 quasars in version 7 of the SDSS quasar catalog (Schneider et al. 2010), all sources with $\mathrm{z}>1.5$ were chosen. The lower redshift limit was adopted to match the approximate lower redshift limit for the heavily absorbed DOGs discovered with Spitzer, for future comparison. (This limit is set because the DOG candidates are found when the 7.8 $\mu \mathrm{m}$ peak for absorbed quasars is within the bandpass for Spitzer surveys at $24 \mu \mathrm{m}$.) The upper redshift limit is the maximum $\mathrm{z} \sim 5$ within the SDSS. The redshift criterion results in 52761 quasars from the SDSS survey. Of these 52761, we select the most luminous 100 as measured by $\nu L_{\nu}(7.8 \mu \mathrm{m})$.

This selection is made using $f_{\nu}(22 \mu \mathrm{m})$ from the WISE Source Catalog. In this catalog, 48651 of the 52761 SDSS quasars have WISE detections listed at some WISE wavelength, assuming a source identification to be correct if the WISE coordinate and SDSS coordinate agree to within $3{ }^{\prime \prime}$. For our uses, the necessary WISE photometry is at $22 \mu \mathrm{m}$, for which we assume a real detection if $f_{\nu}(22 \mu \mathrm{m})>3 \mathrm{mJy}(3 \sigma)$. Using this criterion, 16242 of the SDSS quasars are measured at $22 \mu \mathrm{m}$.

Within the redshift range $1.5<\mathrm{z}<5$, the $f_{\nu}(22 \mu \mathrm{m})$ from WISE corresponds to rest frame wavelengths $3.7 \mu \mathrm{m}<\lambda<8.8 \mu \mathrm{m}$. The observed $f_{\nu}(22 \mu \mathrm{m})$ is transformed to $f_{\nu}(7.8$ $\mu \mathrm{m})$ using the empirical spectral template shown in Figure 6 to scale $f_{\nu}$ between $7.8 \mu \mathrm{m}$ and the rest wavelength corresponding to $22 \mu \mathrm{m}$ observed wavelength. Among the SDSS quasars in the total sample which we use, 12 have also been observed with the IRS3; several of these are discussed in Deo et al. (2011). These 12 SDSS type 1 quasars with $\mathrm{z}>1.5$ are combined with the infrared spectra of 33 AGN with silicate emission from Sargsyan et al. (2011) to produce the template in Figure 6.

Table 3 contains the measurements for this subsample of the 100 most luminous quasars from SDSS and WISE, as determined from $\nu L_{\nu}(7.8 \mu \mathrm{m})$. (Three of these are known gravitationally lensed quasars (Inada et al. 2010); these are included in the Table but not in the plots.)

Virial black hole mass estimates and $L_{b o l}$ are also provided for these SDSS quasars (Shen et al. 2011), derived using the velocity dispersions for the CIV emission line and assuming from Richards et al. (2006) that $L_{b o l}=5.15 \lambda L_{\lambda}(0.30 \mu \mathrm{m})$ for $0.7<\mathrm{z}<1.9$ and $L_{b o l}=3.81 \lambda L_{\lambda}(0.135 \mu \mathrm{m})$ for $\mathrm{z}>1.9$.

\footnotetext{
${ }^{2}$ http://wise2.ipac.caltech.edu/docs/release/allsky/

${ }^{3}$ SDSS $\quad 164016.09+412101.1, \quad 163425.11+404152.5, \quad 161007.11+535814.1, \quad 160950.71+532909.5$, 161238.26+532255.0, 081200.49+402814.3, 140323.39-000606.9, 155855.18+332318.6, 084538.66+342043.6, 030449.85-000813.4, 025905.63+001121.9, and 141546.24+112943.4; spectra are available in CASSIS.
} 


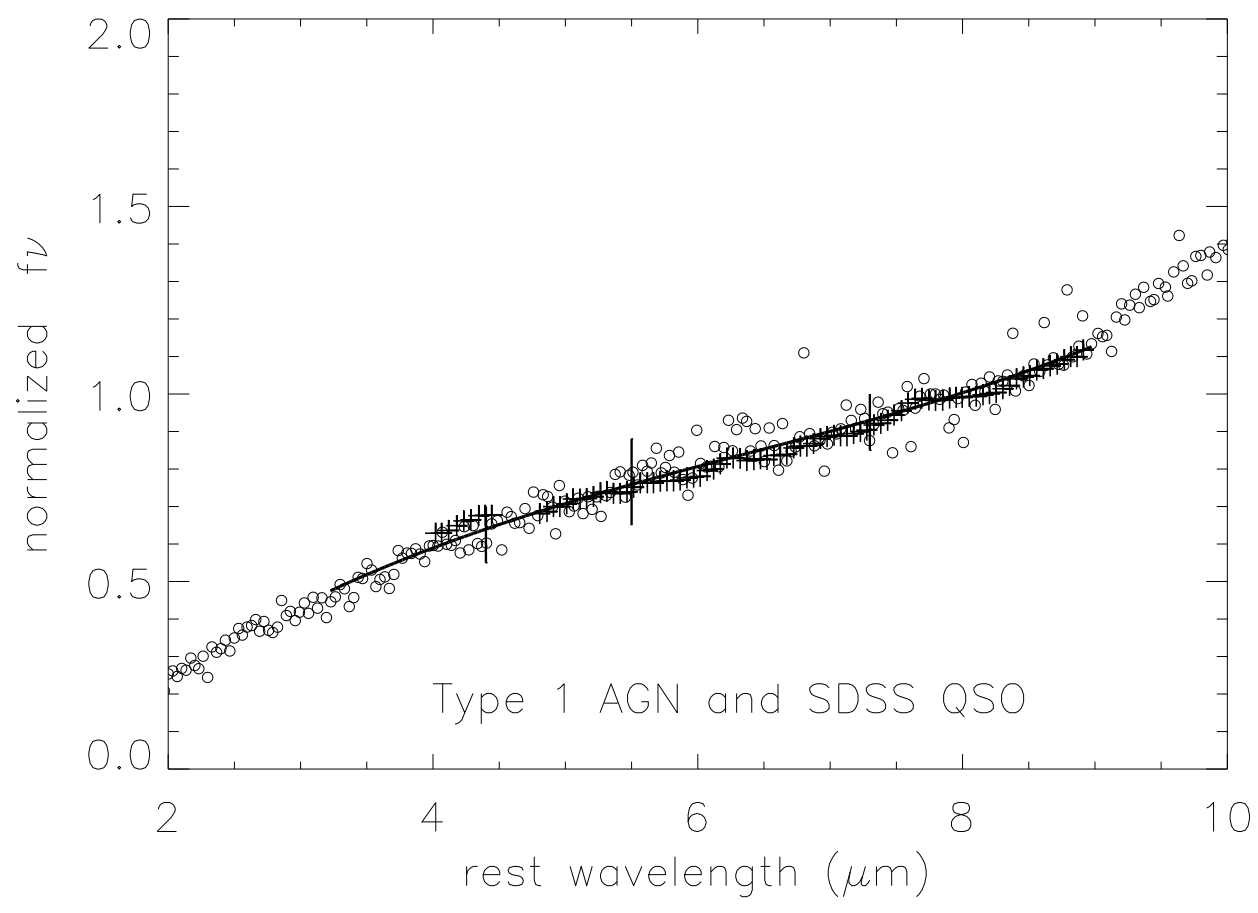

Fig. 6.- Solid line is template used for transforming $f_{\nu}$ (observed $22 \mu \mathrm{m}$ ) to $f_{\nu}$ (rest frame $7.8 \mu \mathrm{m}$ ) for SDSS quasars with WISE photometry at $22 \mu \mathrm{m}$. Circles show median spectrum of 12 SDSS quasars also observed by IRS; crosses show median spectrum of the 33 AGN with silicate emission in Sargsyan et al. (2011). Spectra are normalized to $1 \mathrm{mJy}$ at $7.8 \mu \mathrm{m}$. Vertical error bars show dispersion among overlaid spectra for all of these type 1 AGN and quasars at wavelengths corresponding to observed frame $22 \mu \mathrm{m}$ for $\mathrm{z}=2,3$, and 4 . 
Table 3. Infrared Luminosities for Most Luminous SDSS Quasars

\begin{tabular}{|c|c|c|c|c|c|c|c|c|}
\hline Source & Name & $z^{a}$ & $\begin{array}{c}f_{\nu}(22 \mu \mathrm{m})^{\mathrm{b}} \\
\mathrm{mJy}\end{array}$ & $\begin{array}{c}f_{\nu}(7.8 \mu \mathrm{m})^{\mathrm{c}} \\
\mathrm{mJy}\end{array}$ & $\begin{array}{c}\nu L_{\nu}(7.8 \mu \mathrm{m})^{\mathrm{d}} \\
\log \operatorname{erg~s}^{-1}\end{array}$ & $\begin{array}{c}L_{I R}{ }^{\mathrm{e}} \\
\log \mathrm{L}_{\odot}\end{array}$ & $\begin{array}{c}L_{b o l}{ }^{\mathrm{f}} \\
\log \mathrm{L}_{\odot}\end{array}$ & $\begin{array}{c}\mathrm{BHM}^{\mathrm{g}} \\
\log \mathrm{M}_{\odot}\end{array}$ \\
\hline 1 & SDSS 000654.10-001533.4 & 1.724 & 227.95 & 223.34 & 47.77 & 14.69 & 13.40 & 9.55 \\
\hline 2 & SDSS 000743.78-002432.4 & 1.540 & 104.10 & 95.22 & 47.31 & 14.23 & 13.31 & 9.27 \\
\hline 3 & SDSS 000917.68-001344.8 & 1.533 & 57.42 & 52.39 & 47.04 & 13.96 & 12.45 & 9.11 \\
\hline 4 & SDSS 001022.14-003701.2 & 3.152 & 42.72 & 57.39 & 47.64 & 14.56 & 13.77 & 9.56 \\
\hline 5 & SDSS 002614.69+143105.2 & 3.973 & 8.28 & 12.20 & 47.13 & 14.05 & 13.49 & 9.74 \\
\hline 6 & SDSS 004527.68+143816.1 & 1.992 & 34.50 & 36.76 & 47.10 & 14.02 & 13.85 & 9.42 \\
\hline 7 & SDSS 012403.77+004432.6 & 3.834 & 6.67 & 9.71 & 47.00 & 13.92 & 14.11 & 10.15 \\
\hline 8 & SDSS 012530.85-102739.8 & 3.352 & 8.94 & 12.33 & 47.01 & 13.93 & 13.98 & 9.69 \\
\hline 9 & SDSS 020950.71-000506.4 & 2.828 & 14.84 & 18.98 & 47.08 & 14.00 & 14.32 & 10.18 \\
\hline 10 & SDSS 021646.94-092107.2 & 3.716 & 7.41 & 10.65 & 47.02 & 13.94 & 13.98 & 10.27 \\
\hline 11 & SDSS 024230.65-000029.7 & 2.506 & 24.65 & 29.71 & 47.18 & 14.10 & 12.86 & 9.30 \\
\hline 12 & SDSS 041420.90+060914.2 & 2.632 & 13.55 & 16.74 & 46.97 & 13.89 & 13.62 & 10.28 \\
\hline 13 & SDSS 073502.30+265911.5 & 1.973 & 27.56 & 29.20 & 46.99 & 13.91 & 14.25 & 10.48 \\
\hline 14 & SDSS 074521.78+473436.1 & 3.220 & 10.50 & 14.23 & 47.05 & 13.97 & 14.70 & 9.94 \\
\hline 15 & SDSS 074711.14+273903.3 & 4.154 & 5.64 & 8.45 & 47.00 & 13.92 & 14.16 & 10.46 \\
\hline 16 & SDSS 080117.79+521034.5 & 3.236 & 11.83 & 16.08 & 47.10 & 14.02 & 14.45 & 10.59 \\
\hline 17 & SDSS $081331.28+254503.0^{\mathrm{h}}$ & 1.510 & 91.93 & 83.07 & 47.23 & 14.15 & 14.33 & 9.86 \\
\hline 18 & SDSS 081855.77+095848.0 & 3.674 & 6.83 & 9.78 & 46.98 & 13.90 & 14.07 & 9.98 \\
\hline 19 & SDSS $084631.52+241108.3$ & 4.743 & 4.45 & 6.97 & 47.00 & 13.92 & 13.62 & 10.03 \\
\hline 20 & SDSS 090033.50+421547.0 & 3.290 & 9.34 & 12.78 & 47.01 & 13.93 & 14.51 & 9.82 \\
\hline 21 & SDSS $090334.94+502819.3^{\mathrm{h}}$ & 3.584 & 9.02 & 12.79 & 47.08 & 14.00 & 13.43 & 8.74 \\
\hline 22 & SDSS 090423.37+130920.7 & 2.976 & 23.54 & 30.82 & 47.32 & 14.24 & 14.18 & 9.97 \\
\hline 23 & SDSS 092819.29+534024.1 & 4.390 & 4.60 & 7.03 & 46.96 & 13.88 & 13.56 & 10.05 \\
\hline 24 & SDSS 094140.17+325703.2 & 3.452 & 7.80 & 10.88 & 46.98 & 13.90 & 13.75 & 10.15 \\
\hline 25 & SDSS 094734.19+142116.9 & 3.030 & 9.80 & 12.94 & 46.96 & 13.88 & 14.22 & 10.24 \\
\hline 26 & SDSS 095031.63+432908.4 & 1.771 & 37.59 & 37.41 & 47.01 & 13.93 & 14.10 & 9.75 \\
\hline 27 & SDSS $095841.21+282729.5$ & 3.382 & 15.74 & 21.79 & 47.27 & 14.19 & 14.09 & 10.53 \\
\hline 28 & SDSS 095937.11+131215.4 & 4.056 & 6.29 & 9.34 & 47.03 & 13.95 & 14.40 & 9.72 \\
\hline 29 & SDSS 101336.37+561536.3 & 3.633 & 7.12 & 10.15 & 46.99 & 13.91 & 13.90 & 9.43 \\
\hline 30 & SDSS 101447.18+430030.1 & 3.126 & 9.06 & 12.12 & 46.96 & 13.88 & 14.57 & 10.45 \\
\hline 31 & SDSS $101549.00+002020.0$ & 4.403 & 4.82 & 7.37 & 46.98 & 13.90 & 13.76 & 9.67 \\
\hline 32 & SDSS 102040.61+092254.2 & 3.643 & 7.67 & 10.95 & 47.02 & 13.94 & 13.99 & 10.30 \\
\hline 33 & SDSS $102541.78+245424.2$ & 2.384 & 20.92 & 24.59 & 47.06 & 13.98 & 12.60 & 9.00 \\
\hline 34 & SDSS 102632.97+032950.6 & 3.885 & 6.75 & 9.87 & 47.02 & 13.94 & 13.10 & 9.13 \\
\hline 35 & SDSS 102714.77+354317.4 & 3.109 & 22.01 & 29.39 & 47.34 & 14.26 & 14.44 & 9.91 \\
\hline 36 & SDSS $104846.63+440710.8$ & 4.347 & 4.85 & 7.38 & 46.97 & 13.89 & 13.50 & 9.58 \\
\hline
\end{tabular}


Table 3-Continued

\begin{tabular}{|c|c|c|c|c|c|c|c|c|}
\hline Source & Name & $z^{a}$ & $\begin{array}{c}f_{\nu}(22 \mu \mathrm{m})^{\mathrm{b}} \\
\mathrm{mJy}\end{array}$ & $\begin{array}{c}f_{\nu}(7.8 \mu \mathrm{m})^{\mathrm{c}} \\
\mathrm{mJy}\end{array}$ & $\begin{array}{l}\nu L_{\nu}(7.8 \mu \mathrm{m})^{\mathrm{d}} \\
\log \operatorname{erg~s}^{-1}\end{array}$ & $\begin{array}{c}L_{I R}{ }^{\mathrm{e}} \\
\log \mathrm{L}_{\odot}\end{array}$ & $\begin{array}{c}L_{b o l}{ }^{\mathrm{f}} \\
\log \mathrm{L}_{\odot}\end{array}$ & $\begin{array}{r}\mathrm{BHM}^{\mathrm{g}} \\
\log \mathrm{M}_{\odot}\end{array}$ \\
\hline 37 & SDSS $105122.46+310749.3$ & 4.253 & 4.92 & 7.43 & 46.96 & 13.88 & 13.97 & 9.91 \\
\hline 38 & SDSS $105756.25+455553.0$ & 4.138 & 5.29 & 7.92 & 46.97 & 13.89 & 14.33 & 10.12 \\
\hline 39 & SDSS 110352.74+100403.1 & 3.606 & 7.44 & 10.57 & 47.00 & 13.92 & 13.65 & 9.96 \\
\hline 40 & SDSS 110607.47-173113.5 & 2.572 & 18.29 & 22.34 & 47.08 & 14.00 & 14.02 & 10.25 \\
\hline 41 & SDSS $110610.72+640009.6$ & 2.203 & 24.16 & 27.22 & 47.05 & 13.97 & 14.41 & 10.28 \\
\hline 42 & SDSS 111017.13+193012.5 & 2.497 & 17.58 & 21.15 & 47.03 & 13.95 & 12.24 & 8.07 \\
\hline 43 & SDSS $111038.63+483115.6$ & 2.955 & 17.71 & 23.12 & 47.19 & 14.11 & 14.40 & 10.25 \\
\hline 44 & SDSS $111055.21+430510.0$ & 3.822 & 9.45 & 13.73 & 47.15 & 14.07 & 13.89 & 10.26 \\
\hline 45 & SDSS $111119.10+133603.9$ & 3.481 & 9.49 & 13.30 & 47.07 & 13.99 & 14.35 & 10.34 \\
\hline 46 & SDSS $112258.77+164540.3$ & 3.031 & 15.06 & 19.88 & 47.15 & 14.07 & 13.76 & 10.03 \\
\hline 47 & SDSS 113017.37+073212.9 & 2.659 & 23.98 & 29.77 & 47.23 & 14.15 & 14.16 & 9.95 \\
\hline 48 & SDSS $114117.44+060332.7$ & 3.294 & 9.48 & 12.98 & 47.02 & 13.94 & 12.93 & 8.97 \\
\hline 49 & SDSS 115421.69+025414.0 & 1.671 & 48.73 & 46.86 & 47.06 & 13.98 & 13.01 & 9.34 \\
\hline 50 & SDSS 115747.99+272459.6 & 2.212 & 27.38 & 30.92 & 47.10 & 14.02 & 13.12 & 9.33 \\
\hline 51 & SDSS $115906.52+133737.7$ & 3.984 & 7.09 & 10.47 & 47.06 & 13.98 & 14.29 & 10.66 \\
\hline 52 & SDSS $120006.25+312630.8$ & 2.989 & 11.42 & 14.99 & 47.01 & 13.93 & 14.56 & 10.23 \\
\hline 53 & SDSS 120144.36+011611.6 & 3.233 & 11.44 & 15.53 & 47.09 & 14.01 & 14.19 & 10.30 \\
\hline 54 & SDSS $120147.90+120630.2$ & 3.510 & 8.71 & 12.25 & 47.04 & 13.96 & 14.32 & 9.79 \\
\hline 55 & SDSS $120447.15+330938.7$ & 3.616 & 8.56 & 12.17 & 47.06 & 13.98 & 13.36 & 9.54 \\
\hline 56 & SDSS 121027.62+174108.9 & 3.610 & 9.97 & 14.17 & 47.13 & 14.05 & 13.80 & 10.67 \\
\hline 57 & SDSS $121537.88+022753.3$ & 3.634 & 7.46 & 10.63 & 47.01 & 13.93 & 13.12 & 9.55 \\
\hline 58 & SDSS 121549.81-003432.1 & 2.679 & 18.06 & 22.50 & 47.11 & 14.03 & 13.88 & 9.76 \\
\hline 59 & SDSS $121930.77+494052.2$ & 2.699 & 12.75 & 15.94 & 46.97 & 13.89 & 14.31 & 10.24 \\
\hline 60 & SDSS 122016.87+112628.1 & 1.881 & 47.89 & 49.38 & 47.18 & 14.10 & 13.70 & 8.63 \\
\hline 61 & SDSS $123641.45+655442.1$ & 3.387 & 8.55 & 11.84 & 47.00 & 13.92 & 14.37 & 10.36 \\
\hline 62 & SDSS $123714.60+064759.5$ & 2.781 & 11.75 & 14.90 & 46.96 & 13.88 & 13.53 & 9.81 \\
\hline 63 & SDSS 124551.44+010505.0 & 2.809 & 11.48 & 14.63 & 46.96 & 13.88 & 13.72 & 9.27 \\
\hline 64 & SDSS 124957.23-015928.8 & 3.638 & 9.12 & 13.00 & 47.09 & 14.01 & 14.19 & 10.40 \\
\hline 65 & SDSS 125005.72+263107.5 & 2.048 & 30.98 & 33.53 & 47.08 & 14.00 & 14.58 & 9.80 \\
\hline 66 & SDSS $125050.88+204658.7$ & 3.570 & 7.35 & 10.40 & 46.98 & 13.90 & 13.13 & 10.23 \\
\hline 67 & SDSS 130502.28+052151.1 & 4.086 & 9.47 & 14.10 & 47.21 & 14.13 & 13.87 & 10.43 \\
\hline 68 & SDSS 131011.60+460124.4 & 2.134 & 22.15 & 24.53 & 46.98 & 13.90 & 14.25 & 10.06 \\
\hline 69 & SDSS 132654.96-000530.1 & 3.306 & 10.95 & 15.02 & 47.09 & 14.01 & 12.85 & 8.05 \\
\hline 70 & SDSS 132827.06+581836.8 & 3.139 & 13.95 & 18.71 & 47.15 & 14.07 & 13.53 & 8.60 \\
\hline 71 & SDSS $133335.78+164903.9$ & 2.089 & 23.37 & 25.57 & 46.98 & 13.90 & 14.17 & 9.79 \\
\hline 72 & SDSS $141546.24+112943.4^{\mathrm{h}}$ & 2.560 & 58.16 & 70.87 & 47.58 & 14.50 & 14.28 & 9.33 \\
\hline
\end{tabular}


Table 3-Continued

\begin{tabular}{|c|c|c|c|c|c|c|c|c|}
\hline Source & Name & $z^{\mathrm{a}}$ & $\begin{array}{c}f_{\nu}(22 \mu \mathrm{m})^{\mathrm{b}} \\
\mathrm{mJy}\end{array}$ & $\begin{array}{c}f_{\nu}(7.8 \mu \mathrm{m})^{\mathrm{c}} \\
\mathrm{mJy}\end{array}$ & $\begin{array}{c}\nu L_{\nu}(7.8 \mu \mathrm{m})^{\mathrm{d}} \\
\log \operatorname{erg~s}^{-1}\end{array}$ & $\begin{array}{c}L_{I R} \mathrm{e}^{-} \\
\log \mathrm{L}_{\odot}\end{array}$ & $\begin{array}{c}L_{b o l}^{\mathrm{f}} \\
\log \mathrm{L}_{\odot}\end{array}$ & $\begin{array}{r}\mathrm{BHM}^{\mathrm{g}} \\
\log \mathrm{M}_{\odot}\end{array}$ \\
\hline 73 & SDSS 142123.97+463318.0 & 3.363 & 8.56 & 11.82 & 47.00 & 13.92 & 14.25 & 10.40 \\
\hline 74 & SDSS $142243.02+441721.2$ & 3.545 & 14.99 & 21.16 & 47.29 & 14.21 & 14.00 & 10.45 \\
\hline 75 & SDSS $142656.18+602550.8$ & 3.192 & 22.77 & 30.76 & 47.37 & 14.29 & 14.69 & 10.41 \\
\hline 76 & SDSS $143352.21+022713.9$ & 4.721 & 5.11 & 7.99 & 47.06 & 13.98 & 14.16 & 10.80 \\
\hline 77 & SDSS $143835.95+431459.2$ & 4.611 & 7.47 & 11.60 & 47.21 & 14.13 & 14.41 & 10.38 \\
\hline 78 & SDSS $144105.53+045454.9$ & 2.064 & 26.24 & 28.53 & 47.02 & 13.94 & 13.91 & 10.19 \\
\hline 79 & SDSS $144709.24+103824.5$ & 3.675 & 7.38 & 10.57 & 47.01 & 13.93 & 12.88 & $\ldots$ \\
\hline 80 & SDSS $145125.31+144136.0$ & 3.102 & 13.21 & 17.63 & 47.11 & 14.03 & 13.60 & 9.68 \\
\hline 81 & SDSS $150654.55+522004.7$ & 4.068 & 6.94 & 10.32 & 47.07 & 13.99 & 13.47 & $\cdots$ \\
\hline 82 & SDSS $151352.52+085555.7$ & 2.904 & 25.58 & 33.12 & 47.34 & 14.26 & 14.01 & 10.35 \\
\hline 83 & SDSS $152156.48+520238.5$ & 2.208 & 21.55 & 24.31 & 47.00 & 13.92 & 14.60 & 10.11 \\
\hline 84 & SDSS $153830.55+085517.0$ & 3.551 & 7.96 & 11.25 & 47.01 & 13.93 & 14.46 & 10.02 \\
\hline 85 & SDSS $154446.34+412035.7$ & 3.548 & 7.43 & 10.49 & 46.98 & 13.90 & 13.29 & 10.25 \\
\hline 86 & SDSS $154938.72+124509.1$ & 2.387 & 20.85 & 24.51 & 47.06 & 13.98 & 13.59 & 9.29 \\
\hline 87 & SDSS 155434.17+110950.6 & 2.936 & 18.12 & 23.58 & 47.20 & 14.12 & 12.32 & 8.56 \\
\hline 88 & SDSS $155514.85+100351.3$ & 3.502 & 7.40 & 10.40 & 46.97 & 13.89 & 13.62 & 10.08 \\
\hline 89 & SDSS $155912.34+482819.9$ & 3.423 & 7.79 & 10.84 & 46.97 & 13.89 & 14.15 & 10.30 \\
\hline 90 & SDSS 155952.67+192310.4 & 3.951 & 6.19 & 9.11 & 47.00 & 13.92 & 13.42 & 9.46 \\
\hline 91 & SDSS 162116.92-004250.8 & 3.703 & 7.20 & 10.33 & 47.01 & 13.93 & 14.36 & 9.57 \\
\hline 92 & SDSS $163300.13+362904.8$ & 3.576 & 11.68 & 16.54 & 47.19 & 14.11 & 13.92 & 9.90 \\
\hline 93 & SDSS $163515.49+380804.4$ & 1.813 & 34.79 & 35.11 & 47.00 & 13.92 & 13.78 & 9.53 \\
\hline 94 & SDSS $163909.10+282447.1$ & 3.819 & 20.41 & 29.66 & 47.49 & 14.41 & 14.36 & 10.47 \\
\hline 95 & SDSS $165053.78+250755.4$ & 3.341 & 14.41 & 19.85 & 47.22 & 14.14 & 13.71 & 9.18 \\
\hline 96 & SDSS $170100.60+641209.3$ & 2.735 & 20.94 & 26.36 & 47.19 & 14.11 & 14.67 & 10.36 \\
\hline 97 & SDSS 212329.46-005052.9 & 2.262 & 20.37 & 23.29 & 47.00 & 13.92 & 14.36 & 10.31 \\
\hline 98 & SDSS 223808.07-080842.1 & 3.171 & 9.13 & 12.29 & 46.97 & 13.89 & 13.26 & $\cdots$ \\
\hline 99 & SDSS 234625.66-001600.4 & 3.490 & 9.51 & 13.34 & 47.08 & 14.00 & 14.13 & 10.24 \\
\hline 100 & SDSS 235718.36+004350.4 & 4.364 & 7.42 & 11.32 & 47.16 & 14.08 & 13.40 & 8.69 \\
\hline
\end{tabular}

${ }^{\text {a } O p t i c a l ~ r e d s h i f t ~ f r o m ~ v e r s i o n ~} 7$ of the SDSS quasar catalog (Schneider et al. 2010).

${ }^{\mathrm{b}}$ Observed flux density at $22 \mu \mathrm{m}$ from the WISE All Sky Catalog available at http://wise2.ipac.caltech.edu/docs/release/allsky/. Zero point of $22 \mu \mathrm{m}$ magnitude listed in catalog taken as $8284 \mathrm{mJy}$; typical uncertainties for sources with fluxes listed are $\pm 15 \%$.

${ }^{\mathrm{c}}$ Flux density $f_{\nu}(7.8 \mu \mathrm{m})$ at observed wavelength corresponding to rest wavelength $7.8 \mu \mathrm{m}$, determined by scaling $f_{\nu}$ (observed $22 \mu \mathrm{m}$ ) to $f_{\nu}$ (rest frame $7.8 \mu \mathrm{m}$ ) using tabulated redshift and template spectrum shown in Figure 6 . 
${ }^{\mathrm{d}}$ Rest frame luminosity $\nu L_{\nu}(7.8 \mu \mathrm{m})$ in $\operatorname{erg} \mathrm{s}^{-1}$ determined as $\nu L_{\nu}(7.8 \mu \mathrm{m})=4 \pi \mathrm{D}_{L}^{2}[\nu /(1+\mathrm{z})] f_{\nu}(7.8$ $\mu \mathrm{m})$, for $\nu$ corresponding to $7.8 \mu \mathrm{m}$, taking luminosity distances from Wright (2006): http://www.astro.ucla.edu/ wright/CosmoCalc.html, for $\mathrm{H}_{0}=74 \mathrm{~km} \mathrm{~s}^{-1} \mathrm{Mpc}^{-1}, \Omega_{M}=0.27$ and $\Omega_{\Lambda}=0.73$.

${ }^{\text {e}}$ Total infrared luminosity $L_{I R}$ determined using calibration $\log \left[L_{I R} / \nu L_{\nu}(7.8 \mu \mathrm{m})\right]=0.51$ from Sargsyan et al. (2011) $\left(\log \left[\nu L_{\nu}\left(\mathrm{L}_{\odot}\right)\right]=\log \left[\nu L_{\nu}\left(\operatorname{erg~s}^{-1}\right)\right]-33.59.\right)$

${ }^{\mathrm{f} B o l o m e t r i c ~ l u m i n o s i t y ~} L_{b o l}$ from Shen et al. (2011), adopting a scaling of $L_{b o l}=5.15 \lambda L_{\lambda}(0.30 \mu \mathrm{m})$ for z $<1.9$ and $L_{b o l}=3.81 \lambda L_{\lambda}(0.135 \mu \mathrm{m})$ for $\mathrm{z}>1.9$.

gVirial black hole mass from width of CIV line estimated by Shen et al. (2011).

${ }^{\mathrm{h}}$ Gravitationally lensed source from Inada et al. (2010) and references therein. 


\subsection{Comparisons of Infrared Luminosity to Black Hole Masses and Bolometric Luminosities}

The comparisons of mid-infrared luminosity $\nu L_{\nu}(7.8 \mu \mathrm{m})$ with virial black hole mass are shown in Figure 7. For the local AGN with virial black hole masses from reverberation mapping (crosses and diagonal fit), the luminosity increases linearly with black hole mass (BHM). The linear fit which is shown is $\log \nu L_{\nu}(7.8 \mu \mathrm{m})=37.2+(0.874 \pm 0.11) \log$ BHM for luminosity in erg $\mathrm{s}^{-1}$ and black hole mass in $\mathrm{M}_{\odot}$. The dispersion about the fit in $\log$ $\nu L_{\nu}(7.8 \mu \mathrm{m})$ of \pm 0.5 indicates that this relation is reliable for local type 1 AGN to a factor of 3 .

The dispersion can be caused by many factors: uncertainty in BHM estimates, differences in accretion efficiency, and differences in ratios of mid-infrared luminosity to primary accretion-derived luminosity. The latter factor is already known to have a cosmic dispersion of \pm 0.35 in dex from Figure 4, which compares infrared dust luminosity with intrinsic hard X-ray luminosity. Taking out this dispersion from the \pm 0.5 dispersion in Figure 7 leaves a cosmic dispersion of 0.36 in dex (scatter of a factor of 2.3) for the remaining factors of black hole mass uncertainty and accretion efficiency for the local type 1 AGN used for reverberation mapping.

The fit shown in Figure 7 for local type 1 AGN does not, however, extrapolate to the luminous SDSS/WISE quasars even though all of these are spectroscopically type 1 quasars (based on broad permitted emission lines). In the most extreme case, one quasar is overluminous by a factor of 1000 compared to the local AGN relation between BHM and mid-infrared luminosity! The median values in BHM and luminosity for the quasars show infrared luminosities about a factor of 10 larger than would be expected from the virial black hole mass (or, conversely, a BHM underestimated by a factor of 10 compared to the luminosity). Although this difference is large, it is only about a $2 \sigma$ effect compared to the uncertainties in the virial mass determinations (Peterson et al. 2004; Shen et al. 2011).

There are various reasons that can explain this lack of fit for luminous quasars compared to local AGN. The quasars have been selected as those SDSS quasars that are the most luminous in the mid-infrared as determined from WISE, unlike any selection applied for the local AGN. Selecting for large values of $\nu L_{\nu}(7.8 \mu \mathrm{m})$ favors the type 1 sources with the most extreme hot dust emission. This extreme could arise for a combination of reasons involving the geometry of the dust, including hotter dust because the dust is closer to the AGN or more dust emission because the dust covering factor is greater. These possibilities are considered further in the next section. 


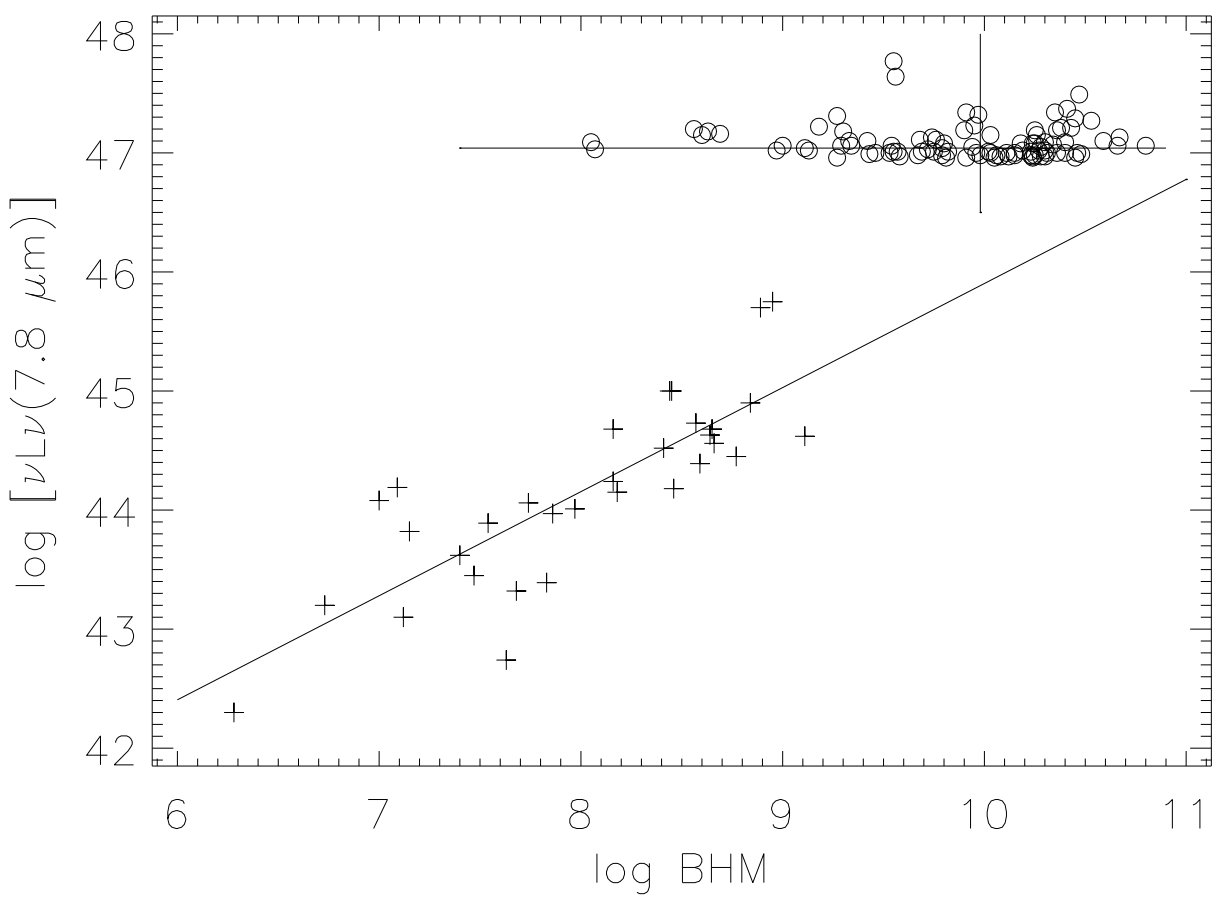

Fig. 7.- Comparison of $\nu L_{\nu}(7.8 \mu \mathrm{m})$ in $\operatorname{erg~s}^{-1}$ with virial black hole mass (solar masses), determined using emission lines from broad line region. Crosses are sources from local AGN with reverberation mapping, determined with Balmer line widths (Peterson et al. 2004). Circles are SDSS quasars, for which BHM estimated from CIV emission line widths (Shen et al. 2011). The diagonal line is the fit to the local AGN. Large cross shows the medians for the SDSS quasars. 


\subsection{Infrared Luminosities compared to Bolometric Luminosities}

The primary bolometric luminosity $L_{b o l}$ arising from the AGN is the fundamental luminosity measurement that is ultimately needed. In the absence of extinction, this $L_{b o l}$ can be scaled from rest frame optical or ultraviolet observations using multiwavelength templates, and such measurements are the primary sources of $L_{b o l}$ (Shen et al. 2011). To understand the uncertainties of such estimates for $L_{b o l}$, we compare to the independent measures of infrared luminosity.

The objective in using an infrared luminosity measure is to achieve an independent measurement of total luminosity that avoids template assumptions and is not sensitive to extinction effects, so that all AGN and quasars can be uniformly compared without concerns about classification or orientation. The simple explanation assumed for the total infrared luminosity $L_{I R}$ is that primary radiation at optical through soft X-ray wavelengths has been absorbed by dust and then reradiated from the heated dust as $L_{I R}$ via the dust continuum emission. If all of the emitting dust is optically thick to the shorter wavelength primary radiation, then the total infrared luminosity emitted must equal the total primary luminosity absorbed. If the dust covering factor is large, therefore, $L_{I R} \sim L_{b o l}$.

The total infrared luminosity $L_{I R}$ is the integrated luminosity from $5 \mu \mathrm{m}$ to $1000 \mu \mathrm{m}$ as defined by Sanders and Mirabel (1996) using infrared fluxes in the photometric bands of the Infrared Astronomical Satellite (IRAS). The $L_{I R}$ have been empirically scaled to $\nu L_{\nu}(7.8$ $\mu \mathrm{m})$ by Sargsyan et al. (2011) using type 1 AGN having measures of both $L_{I R}$ from IRAS and $\nu L_{\nu}(7.8 \mu \mathrm{m})$ from IRS spectra; the scaling is $\log \left[L_{I R} / \nu L_{\nu}(7.8 \mu \mathrm{m})\right]=0.51 \pm 0.21$. We adopt this scaling to estimate $L_{I R}$ for the local AGN in Table 2 and the SDSS/WISE quasars in Table 3.

For the local AGN in Table 2, the $L_{b o l}$ are determined from Richards et al. (2006) as $L_{b o l}$ $=9.26 \lambda L_{\lambda}(0.51 \mu \mathrm{m})$ using the $\lambda L_{\lambda}(0.51 \mu \mathrm{m})$ for the AGN component only (excluding the galactic starlight component) from Bentz et al. (2009). For the SDSS/WISE quasars in Table 3, the $L_{b o l}$ are taken from Shen et al. (2011), adopting a scaling of $L_{b o l}=5.15 \lambda L_{\lambda}(0.30 \mu \mathrm{m})$ for $\mathrm{z}<1.9$ and $L_{b o l}=3.81 \lambda L_{\lambda}(0.135 \mu \mathrm{m})$ for $\mathrm{z}>1.9$. The references cited review the large uncertainties in $L_{b o l}$, especially because no extinction corrections are made to the optical or ultraviolet fluxes to obtain $L_{b o l}$. Our goal is only to illustrate an overall comparison of systematic differences between the independent observational measures $L_{I R}$ and $L_{b o l}$, so we do not examine this comparison for individual sources.

The comparisons of $L_{I R}$ and $L_{b o l}$ are shown in Figure 8. For the AGN sample (crosses

in Figure 8), the median $\log L_{I R} / L_{b o l}=-0.35$. This is the systematic difference that arises when using these two independent measures of total luminosity. If the optically determined 
$L_{b o l}$ are not affected by extinction, i.e. the observed optical luminosity at rest frame 0.51 $\mu \mathrm{m}$ for type $1 \mathrm{AGN}$ shines past dust clouds with no interruption, then the ratio $L_{I R} / L_{b o l}$ is also a measure of the fraction of primary radiation intercepted by dust clouds in directions outside the observer's line of sight. This ratio is equivalent to the covering factor for the dust clouds, corresponding to $45 \%$ with this value of the median. This value of $45 \%$ is similar to the $55 \%$ dust covering factor for BAT AGN determined by silicate absorption in the IRS spectra, discussed in section 2.3 .

The ratio $L_{I R} / L_{b o l}$ for the most infrared-luminous SDSS/WISE quasars (Table 3 and circles in Figure 8) has a median of $\log L_{I R} / L_{b o l}=0.1$, indicating that the optically-derived and infrared-derived measures of total luminosity are nearly the same systematically. This implies a nearly 100\% dust covering factor, which can be explained for these type 1 quasars only if the measured $L_{b o l}$ derives from ultraviolet luminosity that just happens to be observed without extinction through small holes between dust clouds.

The ratio extends to values of $\log L_{I R} / L_{b o l} \sim 2$, however, indicating that for some type 1 quasars, the infrared measures can give much larger total luminosities than the $L_{b o l}$ measures derived from rest frame ultraviolet luminosity. If $\log L_{I R} / L_{b o l}>0$, the simple equating of $L_{I R} / L_{b o l}$ to a dust covering factor cannot apply; this ratio cannot exceed unity when all primary radiation is absorbed and the covering factor is $100 \%$. Cases with $L_{I R}>L_{b o l}$ would anyway be nonsensical within our assumptions, because no rest frame optical or ultraviolet radiation could be observed from which to derive an $L_{b o l}$ if optically thick dust covers $100 \%$ of the source.

Extreme values of $L_{I R}>L_{b o l}$ can be qualitatively explained in part by selection effects that arise from choosing only those type 1 quasars which are most luminous in the midinfrared. There is a one sigma dispersion of \pm 0.2 in the ratio $\log L_{I R} / \nu L_{\nu}(7.8 \mu \mathrm{m})$ calibrated empirically by Sargsyan et al. (2011). Furthermore, section 2.3 showed a dispersion in log of \pm 0.35 for the ratio of hard X-ray flux to mid-infrared $\nu f_{\nu}(7.8 \mu \mathrm{m})$. These two factors reflect the cosmic dispersion in dust temperature and/or intrinsic spectral energy distributions that affect the fundamental relation between $L_{I R}$ and the intrinsic bolometric luminosity of the AGN. The convolved one sigma dispersion of \pm 0.4 can account for much of the apparent excess of $L_{I R}$ for those quasars which are most luminous in $\nu L_{\nu}(7.8 \mu \mathrm{m})$; the most extreme source in $L_{I R} / L_{b o l}$ is only 4 sigma from the median. Variability in the ultraviolet luminosity that is used to measure $L_{b o l}$ could also explain some unusual ratios because the $L_{I R}$ arising from dust reradiation should not be variable.

Furthermore, those quasars with the largest dust covering factors would also be those most likely to have some dust extinction affecting the rest frame ultraviolet that leads to $L_{b o l}$. Measuring $L_{b o l}$ too small because the ultraviolet is not corrected for extinction would 


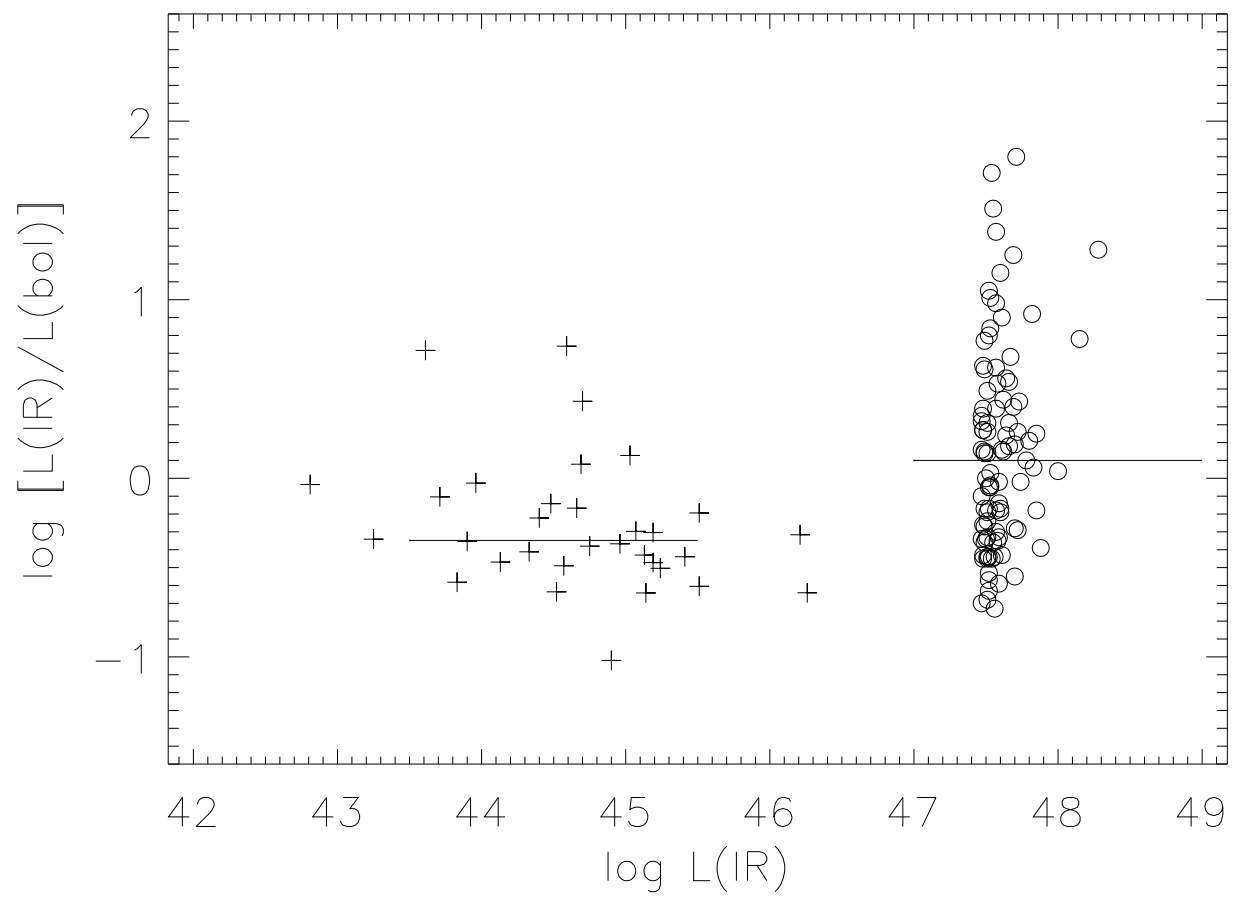

Fig. 8.- Comparison of total infrared luminosities $L_{I R}$ in $\operatorname{erg~s}^{-1}$ to total bolometric luminosity $L_{b o l}$ scaled from observed optical or rest frame ultraviolet luminosity, determined as described in the text. Crosses are local AGN in Table 2 with black hole masses from reverberation mapping. Circles are the 97 SDSS/WISE quasars with $\mathrm{z}>1.5$ which are the most luminous in $\nu L_{\nu}(7.8 \mu \mathrm{m})$, omitting gravitationally lensed sources (Table 3 ). Horizontal lines are medians for the two samples. 
also lead to erroneously large values of $L_{I R} / L_{b o l}$, and this effect is the most straightforward explanation for sources with large values of $L_{I R} / L_{b o l}$.

The most important conclusion from Figure 8 is that infrared measures can give substantially larger values of total luminosity than do $L_{b o l}$ measures derived from rest-frame ultraviolet luminosities, even for type 1 quasars. This means that infrared measures are essential to derive total luminosities not only for the very dusty, optically obscured DOGs, but also for the most luminous optically discovered quasars.

\section{Maximum Luminosities for Dusty Quasars}

A compelling observational challenge is finding the most luminous sources in the universe and understanding how they have changed with cosmic time. What are the maximum luminosities? Has the epoch of maximum luminosity been observed? How do different classes of sources compare in luminosity? Our ultimate goal is to compare luminosities for all sources, including both the classical type 1 quasars and the extremely dusty, optically obscured DOGs. Making this comparison using observed infrared luminosities $\nu L_{\nu}(7.8 \mu \mathrm{m})$ allows a comparison of the most luminous sources among all categories regardless of extinction or discovery technique and independent of assumed multiwavelength spectral templates ("K corrections").

\subsection{Mid Infrared Dust Luminosities of Type 1 AGN and Quasars}

The BAT and SDSS/WISE sources are ideal samples for comparing luminosities of type 1 , silicate emission sources between the local and high redshift universe because both samples arise from large survey areas (BAT covers the full sky, and the SDSS quasar catalog covers $9400 \mathrm{deg}^{2}$ ). The luminosity scaling with redshift of the silicate emission BAT AGN (all sources in Table 1 with silicate strength $>1$ ) and the 97 SDSS/WISE quasars from Table 3 (not including the 3 lensed quasars) is shown in Figure 9.

This Figure displays only the 97 quasars most luminous in $\nu L_{\nu}(7.8 \mu \mathrm{m})$ from the sample of 16242 SDSS quasars in the interval $1.5<\mathrm{z}<5$ with WISE $22 \mu$ m detections. If the sample included all WISE detections $\left(f_{\nu}(22 \mu \mathrm{m})>3 \mathrm{mJy}\right)$, the luminosity detection limit would be as shown by the thick solid line in Figure 9. All of the remaining 16145 SDSS/WISE quasars with $22 \mu \mathrm{m}$ detections would fill in above this limit line.

The results shown in Figure 9 are empirical, observed scaling of luminosities with redshift. These results allow determination of the maximum infrared luminosities which have 


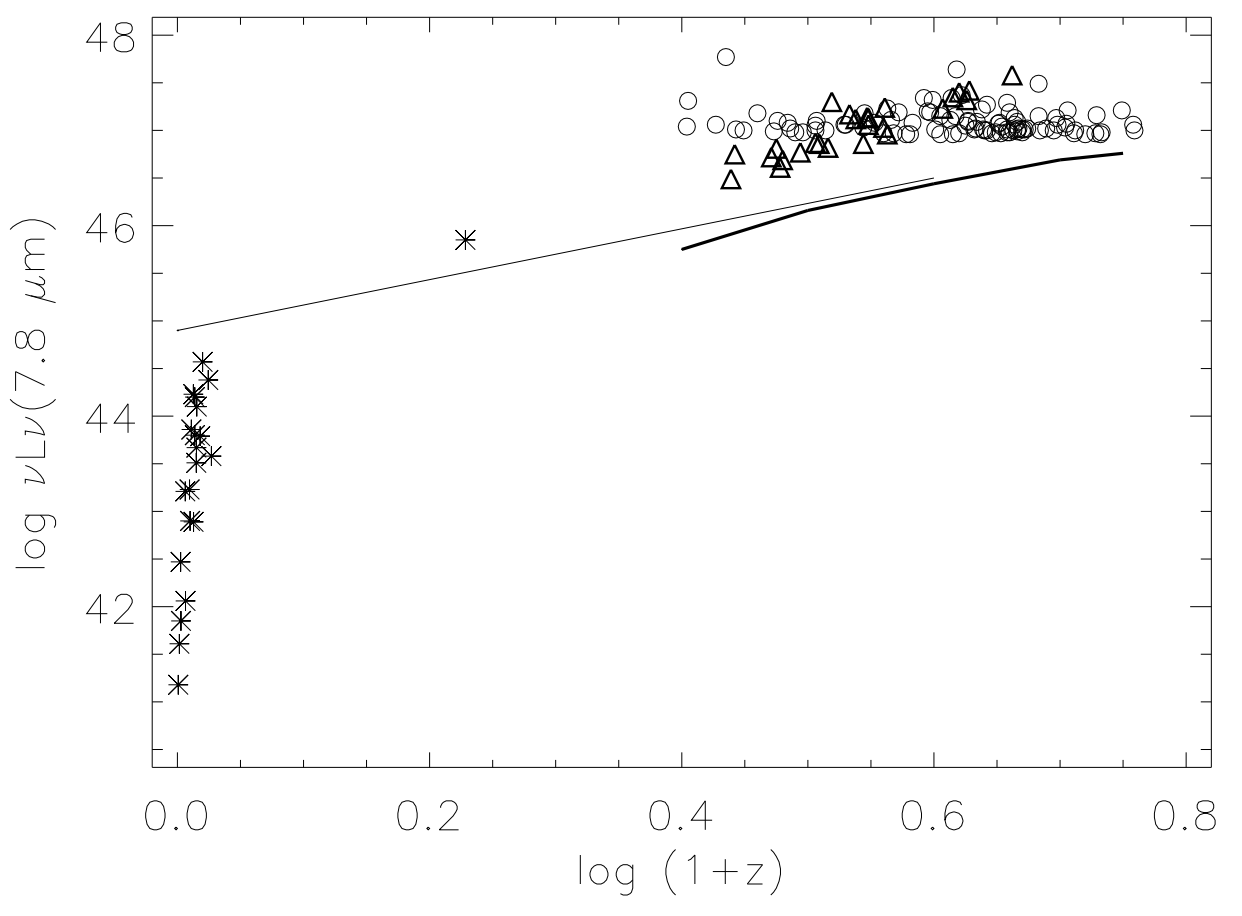

Fig. 9.- Continuum luminosity $\nu L_{\nu}(7.8 \mu \mathrm{m})$ in $\operatorname{erg~s}^{-1}$ compared to redshift for dusty AGN and quasars. Asterisks are silicate emission BAT sources in Table 1 (isolated BAT source in center is 3C 273); circles are SDSS/WISE quasars in Table 3 (excluding 3 lensed sources). Triangles are WISE sources selected for heavy dust obscuration (Wu et al. 2012) scaled to $7.8 \mu \mathrm{m}$ luminosity as described in text. Thin solid line is envelope of most luminous silicate absorbed ULIRGs or DOGs with $0<\mathrm{z}<3$ measured from Spitzer IRS spectra in Weedman and Houck (2009). Thick solid line is faint luminosity limit (lower envelope) found for all SDSS/WISE sources in interval $1.5<\mathrm{z}<5$ using all 16242 SDSS/WISE 22 $\mu \mathrm{m}$ detections brighter than $3 \mathrm{mJy}$ combined with template in Figure $6 . \quad\left(\log \left[\nu L_{\nu}(7.8\right.\right.$ $\left.\left.\mu \mathrm{m})\left(\mathrm{L}_{\odot}\right)\right]=\log \left[\nu L_{\nu}(7.8 \mu \mathrm{m})\left(\mathrm{erg} \mathrm{s}^{-1}\right)\right]-33.59.\right)$ 
been discovered so far and the epoch at which they are found. The scaling of luminosities with redshift does not describe "luminosity evolution" as normally defined, because only the maximum observed luminosities are traced as a function of redshift. Luminosity functions and space densities must be considered in order to determine actual luminosity evolution.

Despite the limitations of a simplified interpretation, several interesting results are obvious in Figure 9. The range of observed luminosities measured with the single observed parameter $\nu L_{\nu}(7.8 \mu \mathrm{m})$ covers a factor exceeding $10^{6}$. (We remind again that the lower luminosity cutoff for SDSS/WISE quasars is artificial; we selected only the 100 most luminous sources.) The most luminous SDSS/WISE quasars have essentially common maximum luminosities for all $\mathrm{z}>1.5$, with maximum luminosities reaching $\log \nu L_{\nu}(7.8 \mu \mathrm{m})=47.5$, corresponding to $\log L_{I R}=48\left(\mathrm{erg} \mathrm{s}^{-1}\right)$ with our empirical $L_{I R} / \nu L_{\nu}(7.8 \mu \mathrm{m})$ calibration, or $L_{I R}=10^{14.4} \mathrm{~L}_{\odot}$ !

There is no indication of any particular redshift at which the luminosity achieves a maximum, and the maximum maintains to the highest observed redshift. A crucial future question for the observer is determining to which redshifts these extremely luminous sources continue to exist.

\subsection{Mid Infrared Dust Luminosities of Optically Obscured Quasars}

One of our primary objectives is to determine if the most luminous quasars have been overlooked in optical surveys because they are DOGs with heavy extinction in the rest frame ultraviolet. A comparison of the most luminous known DOGs with the type 1 quasars is also given in Figure 9.

The envelope of maximum luminosities $\nu L_{\nu}(7.8 \mu \mathrm{m})$ for silicate absorbed local ULIRGs and high redshift DOGs having silicate strength $<0.5$ which have been measured with IRS spectra to $\mathrm{z}=3$ is shown as the line in Figure 9 (Weedman and Houck 2009). Also shown for comparison is a new set of optically faint quasars discovered in the all sky WISE survey using infrared photometric color criteria to select obscured sources (Eisenhardt et al. 2012; $\mathrm{Wu}$ et al. 2012). These sources have optical redshifts $1.7<\mathrm{z}<3.6$ and are interpreted as the most luminous DOGs discovered in the WISE all sky sample.

The comparison shows that the most luminous type 1 SDSS/WISE quasars exceed the dust luminosity of the most luminous, heavily obscured Spitzer DOGs by a factor of $\sim 5$ (at $\mathrm{z}=3$, the redshift limit of currently measured DOGs). Much smaller areas have been surveyed by Spitzer to find DOGs, however, so space density comparisons accounting for survey volumes may infer that equally or more luminous DOGS could be found in wide area 
surveys. Such hyperluminous DOGs should be detected within the WISE survey; the WISE luminosity limit line in Figure 9 for $1.5<\mathrm{z}<5$ illustrates that DOGS which are comparable to the most luminous DOGs already discovered with Spitzer would be readily detectable by WISE. The impossibility of new mid-infrared spectra means, however, that candidate WISE DOGs cannot be unambiguously compared to the Spitzer DOGs whose redshifts arise from infrared spectra and whose identification is completely independent of optical measurements.

Because the infrared spectra of these WISE DOGs are not known, there is no confident template with which to relate observed frame $22 \mu \mathrm{m}$ to rest frame $7.8 \mu \mathrm{m}$. Mid-infrared spectra could range from the type 1 template in Figure 6 to the absorbed AGN spectrum in Figure 1. A larger $7.8 \mu \mathrm{m}$ luminosity arises if the silicate absorbed spectrum in Figure 1 is the template because observed-frame $22 \mu \mathrm{m}$ observations then require larger corrections to rest-frame $7.8 \mu \mathrm{m}$ (observed-frame $22 \mu \mathrm{m}$ is $<7.8 \mu \mathrm{m}$ rest frame for all $\mathrm{z}>1.8$ ). We estimate in this way the maximum luminosities for the 26 WISE DOGs in Wu et al. (2012) with redshifts and $22 \mu \mathrm{m}$ photometry. These are the luminosities shown in Figure 9 (the most luminous with these assumptions is W0410-0913).

Overall, the most luminous of these WISE DOGs are similar to the most luminous SDSS/WISE quasars in $\nu L_{\nu}(7.8 \mu \mathrm{m})$, but the DOGs must be more obscured because they are too faint optically to be within the SDSS. Many WISE DOGs are optically brighter than the Spitzer DOGs, however, indicating that they are not as obscured when judged by the infrared/optical flux ratios. In the absence of infrared spectra, we cannot conclude if the WISE DOGs have silicate absorption as strong as the Spitzer DOGs, or represent an intermediate category with less silicate absorption. An indication that the WISE DOGs and Spitzer DOGs represent similar populations of obscured AGN is the similar slope for increasing luminosity with increasing redshift, differing only that the WISE sources are systematically more luminous by about a factor of 3 (0.5 in dex) compared to the most luminous Spitzer sources.

Figure 9 summarizes what is known observationally regarding the most infrared luminous quasars. At present, the optically discovered SDSS/WISE quasars remain the most luminous dusty sources known in the universe. We find no evidence of hyperluminous, obscured quasars whose infrared luminosities significantly exceed the infrared luminosities of optically discovered quasars. This result addresses only the question of maximum quasar luminosities. It is also important to compare the total space densities of optically discoverable, less obscured quasars $(\sim$ type 1$)$ to the optically faint, heavily obscured quasars $(\sim$ type 2 ), and to understand evolution for these different populations. The similar trends among both WISE DOGs and Spitzer DOGs for maximum luminosity to increase with redshift, contrasted to constant maximum luminosity for type 1 quasars, is an observational hint that 
dusty populations may become more important at high redshift.

There are no wide area samples of heavily obscured DOGs with spectroscopic infrared classifications, but there are limited area samples of heavily absorbed DOGs with Spitzer IRS spectra. An initial summary of these silicate absorbed quasars was in Weedman and Houck (2009); we have now identified 74 sources in CASSIS with $1.5<\mathrm{z}<3.35$ and silicate strength $\lesssim 0.5$. In a future analysis, we will compare space densities at similar redshifts among the heavily absorbed DOGs already discovered by Spitzer, the all sky WISE DOGs, and the SDSS/WISE type 1 quasars. This will allow more quantitative conclusions about the dusty fraction of the most luminous quasars and whether dust content evolves with redshift. Such an analysis will also allow predictions of how dusty quasars at even higher redshifts should appear in far infrared and submillimeter surveys, depending on various assumptions of how the luminosity function at $\mathrm{z} \sim 2.5$ extrapolates to higher redshifts.

\section{Summary and Conclusions}

Mid-infrared spectroscopic results from Spitzer IRS spectra are given for 125 hard X-ray AGN (14-195 keV) from the Swift BAT sample, calibrating strength of the $9.7 \mu \mathrm{m}$ silicate absorption or emission feature compared to X-ray luminosity and optical classification. The presence of silicate emission or absorption defines an infrared AGN classification describing whether or not AGN are observed through dust clouds, and the quantitative silicate strength is found to be consistent overall with optical classifications from type 1 through type 2 . The silicate classification indicates that $55 \%$ of the BAT AGN are observed through dust clouds.

From the BAT sample, the mid-infrared dust continuum luminosity is found to be an excellent indicator of intrinsic AGN luminosity, scaling closely with the hard X-ray luminosity, $\log \left[\nu L_{\nu}(7.8 \mu \mathrm{m}) / L(\mathrm{X})\right]=-0.31 \pm 0.35$. This scaling is independent of classification determined from silicate strength, demonstrating that $\nu L_{\nu}(7.8 \mu \mathrm{m})$ is a consistent indicator of luminosity that applies equally to unobscured, type 1 AGN as well as highly obscured type 2 AGN.

IRS spectra are also presented for 32 AGN with black hole mass measures from reverberation mapping. Mid-infrared dust luminosity scales closely with black hole mass, as log $\nu L_{\nu}(7.8 \mu \mathrm{m})=37.2( \pm 0.5)+0.87 \log \mathrm{BHM}$ for luminosity in erg $\mathrm{s}^{-1}$ and $\mathrm{BHM}$ in $\mathrm{M}_{\odot}$.

The 100 most luminous type 1 quasars as measured in $\nu L_{\nu}(7.8 \mu \mathrm{m})$ are found by comparing SDSS optically discovered quasars with WISE photometry at $22 \mu \mathrm{m}$, using an empirical template determined from IRS spectra of type 1 sources for scaling to rest frame $7.8 \mu \mathrm{m}$. The most luminous SDSS/WISE quasars have similar maximum infrared luminosities for all 
$1.5<\mathrm{z}<5$, reaching $\log \nu L_{\nu}(7.8 \mu \mathrm{m})=47.5\left(\mathrm{erg} \mathrm{s}^{-1}\right)$. With our empirical scaling for type 1 sources that $\log L_{I R} / \nu L_{\nu}(7.8 \mu \mathrm{m})=0.51$, for $L_{I R}$ the total infrared luminosity from 5 $\mu \mathrm{m}$ to $1000 \mu \mathrm{m}$, this maximum is $L_{I R}=10^{14.4} \mathrm{~L}_{\odot}$. There is no indication of any particular redshift for $\mathrm{z}<5$ at which the infrared luminosity is maximum.

For the black hole AGN sample and SDSS/WISE quasars, the bolometric luminosities $L_{b o l}$ estimated by scaling from rest frame optical or ultraviolet luminosities are compared to total infrared luminosities $L_{I R}$. For the local AGN, the median $\log L_{I R} / L_{b o l}=-0.35$, indicating a covering factor of $45 \%$ for the dust clouds that absorb intrinsic $L_{b o l}$ and reemit as $L_{I R}$. For the SDSS/WISE quasars deliberately selected as the most luminous infrared sources, the median $\log L_{I R} / L_{b o l}=0.1$, with extremes reaching values of $\sim 2$, indicating that ultraviolet-derived $L_{b o l}$ can be seriously underestimated even for type 1 quasars.

The optically discovered SDSS/WISE type 1 quasars are the most luminous dusty sources currently known in the universe. We find no evidence of hyperluminous, obscured quasars whose infrared luminosities significantly exceed the infrared luminosities of optically discovered quasars. The most luminous type 1 quasars exceed the dust luminosity of the most luminous, heavily obscured quasars (the DOGs found in limited area surveys with Spitzer) by a factor of $\sim 5$ and have comparable maximum luminosities to the recently discovered population of optically faint, dusty quasars from the all sky WISE survey.

This work is based in part on observations made with the Spitzer Space Telescope, which is operated by the Jet Propulsion Laboratory, California Institute of Technology, funded by the National Aeronautics and Space Administration. Support for this work by the IRS GTO team at Cornell University was provided by NASA through Contracts issued by JPL/Caltech.

This publication makes use of data products from the Wide-field Infrared Survey Explorer, which is a joint project of the University of California, Los Angeles, and JPL/Caltech, funded by NASA.

We also acknowledge data products from the SDSS. Funding for the SDSS has been provided by the Alfred P. Sloan Foundation, the Participating Institutions, the National Science Foundation, the U.S. Department of Energy, NASA, the Japanese Monbukagakusho, the Max Planck Society, and the Higher Education Funding Council for England. The SDSS is managed by the Astrophysical Research Consortium for the Participating Institutions: the American Museum of Natural History, Astrophysical Institute Potsdam, University of Basel, University of Cambridge, Case Western Reserve University, University of Chicago, Drexel University, Fermilab, the Institute for Advanced Study, the Japan Participation Group, Johns Hopkins University, the Joint Institute for Nuclear Astrophysics, the Kavli Institute 
for Particle Astrophysics and Cosmology, the Korean Scientist Group, the Chinese Academy of Sciences (LAMOST), Los Alamos National Laboratory, the Max-Planck-Institute for Astronomy (MPIA), the Max-Planck-Institute for Astrophysics (MPA), New Mexico State University, Ohio State University, University of Pittsburgh, University of Portsmouth, Princeton University, the United States Naval Observatory, and the University of Washington.

\section{REFERENCES}

Alexander D. M. et al., 2003, AJ, 126, 539

Antonucci, R.R.J. and Miller, J.S. 1985, ApJ, 297, 621

Bentz, M., Peterson, B. M., Netzer, H., Pogge, R. W., and Vestergaard, M. 2009, ApJ, 697, 160

Boksenberg, A., Carswell, R. F., Allen, D. A., Fosbury, R. A. E., Penston, M. V., and Sargent, W. L. W. 1977, MNRAS, 178, 451

Bussmann, R. S. et al. 2009, ApJ, 693, 750

Brand, K. et al. 2006, ApJ, 644, 143

Brown, M. et al. 2006,ApJ, 638, 88

Chapman, S., Blain, A., Smail, I., and Ivison, R. 2005, ApJ, 622, 77

Croom, S. M., Smith, R. J., Boyle, B. J., Shanks, T., Miller, L., Outram, P. J., and Loaring, N. S. 2004, MNRAS, 349, 1397

Deo, R. P., Richards, G. T., Nikutta, R., Elitzur, M., Gallagher, S. C., Ivezic, Z., and Hines, D. 2011, ApJ, 729, 108.

Dey, A. et al. 2008, ApJ, 677, 943

Donley, J. L., Rieke, G. H., Prez-Gonzlez, P. G., Rigby, J. R., and Alonso-Herrero, A. 2007, ApJ, 660, 167

Draine, B. T. 2003a, ApJ, 598, 1017

Draine, B. T. 2003a, ApJ, 598, 1026

Eisenhardt, P. R. M. et al. 2012, ApJ, 755, 173 
Elitzur, M. 2012, ApJ, 747, 33

Fan, X., et al. 2004, AJ, 128, 515

Fabian, A. C. 1999, MNRAS, 308, L39

Fiore, F. et al. 2008, ApJ, 672, 94

Gallagher, S. C. et al. 2005, ApJ, 633, 71

Gehrels, N. et al. 2004, ApJ, 611, 1005

Genzel, R. et al. 1998, ApJ, 498, 579

Gilli, R., Comastri, A., and Hasinger, G. 2007, A\&A, 463, 79

Gunn, J. E., et al. 1998, AJ, 116, 3040

Hao, L. et al. 2005, ApJ, 625, L75

Hao, L. et al. 2007, ApJ, 655, L77

Hickox, R. C. et al. 2007, ApJ671, 1365

Higdon, S.J.U., et al. 2004, PASP, 116, 975

Houck, J. R. et al. 2004, ApJS, 154, 18

Houck, J.R. et al. 2005, ApJ, 622, L105

Imanishi, M., Dudley, C. C., Maiolino, R., Maloney, P. R., Nakagawa, T., and Risaliti, G. 2007, ApJ, 171, 72

Inada, N. et al. 2010, AJ, 140, 403

Lebouteiller, V., Bernard-Salas, H., Sloan, G. C., and Barry, D. J. 2010, PASP, 122, 231

Lebouteiller, V., Barry, D. J., Bernard-Salas, H., Sloan, G. C., Houck, J. R., and Weedman, D. W. 2010, ApJS, 196, 8

Levenson, N. A., Sirocky, M. M., Hao, L., Spoon, H. W. W., Marshall, J. A., Elitzur, M., and Houck, J. R. 2007, ApJ, 654, 45

Madau, P., Pozzetti, L., and Dickinson, M. 1998, ApJ, 498, 106

Maiolino, R. and Rieke, G. H. 1995, ApJ, 454, 95. 
Martinez-Sansigre, A., et al. 2006, MNRAS, 370, 1479

Peterson, B. M. et al. 2004, ApJ, 613, 682

Ramos-Almeida, C. et al. 2011, ApJ, 731, 92

Reddy, N. A. and Steidel, C. C. 2009, ApJ, 692, 778

Richards, G. T. et al. 2006, ApJS, 166, 470

Rigby, J. R., Diamond-Stanic, A. M., and Aniano, G. 2009, ApJ, 700, 1878

Sajina, A., Yan, L., Armus, L., Choi, P., Fadda, D., Helou, G., and Spoon, H. 2007, ApJ, 664,713

Sanders, D. B., and Mirabel, I. F. 1996, ARA\&A, 34, 749

Sargsyan, L., Weedman, D., Lebouteiller, V., Houck, J., Barry, D., Hovhannisyan, A., and Mickaelian, A. 2011, ApJ, 730, 19

Sargsyan, L. et al 2012, ApJ, 755, 171.

Schneider, D. P. et al. 2010, AJ, 139, 2360

Setti, G. and Woltjer, L. 1989, A\&A, 224, L21

Shen, Y. et al. 2011, ApJS, 194, 45

Shi, Y. et al. 2006, ApJ, 653, 127

Sirocky, M. M., Levenson, N. A., Elitzur, M., Spoon, H. W. W., and Armus, L. 2008, ApJ, 678,729

Soifer, B. T., Neugebauer, G., and Houck, J. R. 1987, ARA\&A, 25, 187

Spoon, H. W. W. et al. 2007, ApJ, 654, L49

Thompson, G. D., Levenson, N. A., Uddin, S. A. and Sirocky, M. M. 2009, ApJ, 697, 182

Tueller, J. et al. 2010, ApJS, 186, 378

Weaver, K. A. et al. 2010, ApJ, 716, 1151

Weedman, D.W. and Houck, J.R. 2009, ApJ, 698, 1682

Willott, C. J., Rawlings, S., Blundell, K. M., and Lacy, M. 2000, MNRAS, 316, 449 
Wright, E. L. 2006, PASP, 118, 1711

Wright, E. L. et al. 2010, AJ, 140, 1868

Wu, J. et al. 2012, ApJ, 756, 96

Yan, L. et al. 2007, ApJ, 658, 778 